\title{
Information-Theoretic Measures for Objective Evaluation of Classifications
}

\author{
Bao-Gang $\mathrm{Hu}^{*, \mathrm{a}, \mathrm{b}}$, Ran $\mathrm{He}^{\mathrm{a}}$, XiaoTong Yuan ${ }^{\mathrm{c}}$ \\ ${ }^{a}$ NLPR/LIAMA, Institute of Automation, Chinese Academy of Sciences, Beijing 100190, China \\ ${ }^{b}$ Beijing Graduate School, Chinese Academy of Sciences, Beijing 100190, China. \\ ${ }^{c}$ Department of Electronic and Computer Engineering, National University of Singapore, Singapore.
}

\begin{abstract}
This work presents a systematic study of objective evaluations of abstaining classifications using Information-Theoretic Measures (ITMs). First, we define objective measures for which they do not depend on any free parameter. This definition provides technical simplicity for examining "objectivity" or "subjectivity" directly to classification evaluations. Second, we propose twenty four normalized ITMs, derived from either mutual information, divergence, or crossentropy, for investigation. Contrary to conventional performance measures that apply empirical formulas based on users' intuitions or preferences, the ITMs are theoretically more sound for realizing objective evaluations of classifications. We apply them to distinguish "error types" and "reject types" in binary classifications without the need for input data of cost terms. Third, to better understand and select the ITMs, we suggest three desirable features for classification assessment measures, which appear more crucial and appealing from the viewpoint of classification applications. Using these features as "meta-measures", we can reveal the advantages and limitations of ITMs from a higher level of evaluation knowledge. Numerical examples are given to corroborate our claims and compare the differences among the proposed measures. The best measure is selected in terms of the meta-measures, and its specific properties regarding error types and reject types are analytically derived.
\end{abstract}

Key words: Abstaining classifications, error types, reject types, entropy, similarity, objectivity

\section{Introduction}

The selection of evaluation measures for classifications has received increasing attentions from researchers on various application fields [1] [2][3][4][5] [6][7]. It is well known that evaluation measures, or criteria, have a substantial impact on the quality of classification performance. The problem of how to select evaluation measures for the overall quality of classifications is difficult, and there appears no universal answer to this.

Up to now, various types of evaluation measures have been used in classification applications. Taking a binary classification as an example, more than thirty metrics have been applied for assessing the quality of classifications and their algorithms as given in Table 1 of Lavesson and Davidsson's paper [5]. Most of the metrics listed in this table can be considered a type of performance-based measures. In practice, other types of evaluation measures, such as Information-Theoretic Measures (ITMs), have also commonly been used in machine learning [8][9]. The typical information-based measure used in classifications is the cross entropy [10]. In a recent work [11], Hu and Wang derived an analytical formula of the Shannon-based mutual information measure with respect to a confusion matrix. Significant benefits were derived from the measure, such as its generality even for cases of classifications with a reject option, and its objectivity in naturally balancing performance-based measures that may conflict with one another (such as precision and recall). The objectivity was achieved from the perspective that an information-based measure does not require knowledge of cost terms in evaluating classifications. This advantage is particularly important in studies of abstaining classifications [12][4] and cost sensitive learning [13][14], where cost terms may be required as input

${ }^{*}$ Corresponding author. Address: NLPR/LIAMA, Institute of Automation, Chinese Academy of Sciences, Beijing 100190, China. Tel.: +8610-62647318, Fax: +86-10-62647458.

Email address: hubg@nlpr.ia.ac.cn (Bao-Gang Hu) 
data for evaluations. Generally, if no cost terms are assigned to evaluations, it implies that the zero-one cost functions are applied [15]. In such situations, classification evaluations without a reject option may still be applicable and useful in class-balanced datasets. Problematic, or unreasonable, results will be obtained for evaluations in situations where classes are highly skewed in the datasets [3] if no specific cost terms are given.

In this work, for simplifying discussions, we distinguish, or decouple, two study goals in evaluation studies, namely, evaluation of classifiers and evaluation of classifications. The former goal concerns more about evaluation of algorithms in which classifiers applied. From this evaluation, designers or users can select the best classifier. The latter goal is to evaluate classification results without concerning which classifier is applied. This evaluation aims more on result comparisons or measure comparisons. One typical example was demonstrated by Mackay [16] for highlighting the difficulty in classification evaluations. He showed two specific confusion matrices, $\mathbf{C}_{D}$ and $\mathbf{C}_{E}$, in binary classifications with a reject option:

$$
\mathbf{C}_{D}=\left[\begin{array}{ccc}
74 & 6 & 10 \\
0 & 9 & 1
\end{array}\right], \quad \mathbf{C}_{E}=\left[\begin{array}{ccc}
78 & 6 & 6 \\
0 & 5 & 5
\end{array}\right], \quad \text { with } \quad \mathbf{C}=\left[\begin{array}{ccc}
T N & F P & R N \\
F N & T P & R P
\end{array}\right],
$$

where the confusion matrix is defined as $\mathbf{C}$ in eq. (1), and " $T N "$ ", "TP", " $F N "$, " $F P "$, " $R N$ ", "RP" represent "true negative", "true positive", "false negative", "false positive", "reject negative", "reject positive", respectively. For the given data, users may ask "which measures will be proper for ranking them". If directly applying "True Positive RateFalse Positive Rate" curve (also called ROC) or "Precision-Recall" curve, one may conclude that the performance of $\mathbf{C}_{E}$ is better than that of $\mathbf{C}_{D}$. This conclusion is proper since the two sets of data share the same reject rate $(=11 \%)$. Generally, "Error-Reject" curve is mostly adopted in abstaining classifications. Based on this evaluation approach, one may consider the performances of two classifications have no difference because they show the same error rate $(=6 \%)$ and reject rate. Mackay [16] first suggested applying mutual-information based measure in ranking classifications, and through which $\mathrm{Hu}$ and Wang (referring to M5-M6 in Table 3, [11]) observed that $\mathbf{C}_{D}$ is better than $\mathbf{C}_{E}$. If reviewing the two matrices carefully with respect to imbalanced classes, one may agree with the observation because the small class in $\mathbf{C}_{D}$ receives more correct classifications than that in $\mathbf{C}_{E}$.

We consider the example designed by Mackay [16] is quite stimulating for study of abstaining classification evaluations. The implications of the example form the motivations of the present work on addressing three related open problems, which are generally overlooked in the study of classification evaluations as follows:

I. How to define "proper" measures in terms of high-level knowledge for abstaining classification evaluations?

II. How to conduct an objective evaluation of classifications without using cost terms?

III. How to distinct or rank "error types" and "reject types" in classification evaluations?

Conventional binary classifications usually distinguish two types of misclassification errors [15][16] if they result in different losses in applications. For example, in medical applications, "Type I Error" (or "false positive") can be an error of misclassifying a healthy person to be abnormal, such as cancer. On the contrary, "Type II Error"(or "false negative") is an error where cancer is not detected in a patient. Therefore, "Type II Error" is more costly than "Type I Error". Based on the same reason for identifying "error types" in binary classifications, there is a need for considering "reject types" if a reject option is applied. Of the existing measures, we consider information-theoretic measures to be most promising in providing "objectivity" in classification evaluations. A detailed discussion on the definition of "objectivity" is given in Section 3. This work is an extension of our previous study [11]. However, the work aims at a systematic investigation of information measures with specific focus on "error types" and "reject types". The main contribution of the work is derived from the following three aspects:

I. We define the "proper" features, also called "meta-measures", for selecting candidate measures in the context of abstaining classification evaluations. These features will assist users in understanding advantages and limitations of evaluation measures from a higher level of knowledge.

II. We examine most of the existing information measures in a systematic investigation of "error types" and "reject types" for objective evaluations. We hope that the more than twenty measures investigated are able to enrich the current bank of classification evaluation measures. For the best measure in terms of the meta-measures, we present a theoretical confirmation of its desirable properties regarding error types and reject types. 
III. We reveal the intrinsic shortcomings of information measures in evaluations. The discussions are intended to be applicable to a wider range of classification problems, such as similarity ranking. In addition, we are able to employ the measures reasonably in interpreting classification results.

To address classification evaluations with a reject option, we assume that the only basic data available for classification evaluations is a confusion matrix, without input data of cost terms. The rest of this letter is organized as follows. In Section 2, we present related work for the selection of evaluation measures. For seeking "proper" measures, we propose several desirable features in the context of classifications in Section 3. Three groups of normalized information measures are proposed along with their intrinsic shortcomings in Sections 4 to 6, respectively. Several numerical examples, together with discussions, are given in Section 7. Finally, in Section 8 we conclude the work.

\section{Related Work}

In classification evaluations, a measure based on classification accuracy has traditionally been used with some success in numerous cases [15]. This measure, however, may suffer serious problems in reaching intuitively reasonable results from certain special cases of real-world classification problems [3]. The main reason for this is that a single measure of accuracy does not take into account error types.

To overcome the problems of accuracy measures, researchers have developed many sophisticated approaches for classification assessment[17][18]. Among these, two commonly-used approaches are ROC (Receiver Operating Characteristic) curves and AUC (Area under Curve) measures [1][19]. ROC curves provide users with a very fast evaluation approach via visual inspections, but this is only applicable in limited cases with specific curve forms (for example, when one curve is completely above the other). AUC measures are more generic for ranking classifications without constraints on curve forms. In a study of binary classifications, a formal proof was given by Ling et al. [1] showing that AUC is a better measure than accuracy from the definitions of both statistical consistency and discriminancy. Sophisticated AUC measures were reported recently for improving robustness [6] and coherency [7] of classifiers. Drummond and Holte [20] proposed a visualization technique called "Cost Curve", which is able to take into account of cost terms for showing confidence intervals on classifier's performance. Japkowicz [3] presented convincing examples showing the shortcomings of the existing evaluation methods, including accuracy, precision vs. recall, and ROC techniques. The findings from the examples further confirmed the need for methods using measurebased functions [21]. The main idea behind measure-based functions is to form a single function with respect to a weighted summation of multiple measures. The measure function is able to balance a trade-off among the conflicting measures, such as precision and recall. However, the main difficulty arises in the selection of balancing weights for the measures [5]. In most cases, users rely on their preferences and experiences in assigning the weights, which imposes a strong degree of subjectivity on the evaluation results.

Classification evaluations become more complicated if a classifier abstains from making a prediction when the outcome is considered unreliable for a specific sample. In this case, an extra class, known as the "reject" or "unknown" class, is added to the classification. In recent years, the study of abstaining classifiers has received much attention [22][23][12][4][24]. With complete data of a full cost matrix, they were able to assess the classifications. If one term of the cost matrix was missing, such as on a reject cost term, the approaches for classification evaluations generally failed. Moreover, because in most situations the cost terms are given by users, this approach is basically a subjective evaluation in applications. Vanderlooy et al. [25] further investigated the ROC isometrics approach which does not rely on information from a cost matrix. This approach, however, is only applicable to binary classification problems.

A promising study of objective evaluations of classifications is attributed to the introduction of information theory. Kvalseth [26] and Wickens [27] derived normalized mutual information (NMI) measures in relation to a contingency table. Further pioneering studies on the classification problems were conducted by Finn [28] and Forbes [29]. Forbes [29] discussed the problem that NMI does not share a monotonic property with the other performance measures, such as accuracy or F-measure. Several different definitions for information measures have been reported in studies of classification assessment, such as information scores by Kononenko and Bratko [30] and KL divergence by Nishii and Tanaka [31]. Yao, et al. [8] and Tan, et al. [32] summarized many useful information measures for studies of associations and attribute importance. Significant efforts were made on discussing the desired properties of evaluation measures [32]. Principe, et al. [9] proposed a framework of information theoretic learning (ITL) that included supervised learning as in classifications. Within this framework, the learning criteria were the mutual information 
defined from the Shannon and Renyi entropies. Two quadratic divergences, namely, the Euclidean and CauchySchwartz distances were also included.

From the perspective of information theory, Wang and $\mathrm{Hu}$ [33] derived for the first time the nonlinear relations between mutual information and the conventional performance measures (accuracy, recall and precision) for binary classification problems. They [11] extended the investigation into abstaining classification evaluations for multiple classes. Their method was based solely on the confusion matrix. For gaining the theoretical properties, they derived the extremum theorems concerning mutual information measures. One of the important findings from the local minimum theorem is the theoretic revelation of the non-monotonic property of mutual information measures with respect to the diagonal terms of a confusion matrix. This property may cause irrational evaluation results from some data in classifications. They confirmed this problem by examining specific numerical examples. Theoretical investigations are still missed for other information measures, such as divergence-based and cross-entropy based ones.

\section{Objective Evaluations and Meta-Measures}

This work focuses on objective evaluations of classifications. While Berger [34] stressed four points from a philosophical position for supporting objective Bayesian analysis, it seems that few studies in the literature address the "objectivity" issue in the study of classification evaluations. Some researchers [32] may call their measures to be objective ones without defining them formally. Considering that "objectivity" is a more philosophical concept without a well accepted definition, we propose a scheme for defining "objective evaluations" from the viewpoint of practical implementation and examination.

Definition 1. Objective evaluations and measures. An objective evaluation is an assessment expressed by a function that does not contain any free parameter. This function is called an objective measure.

Remark 1. When a free parameter is used to define a measure, it usually carries a certain degree of subjectivity in evaluations. Therefore, according to this definition, a measure based on cost terms [15] as free parameters does not lead to an objective evaluation. Definition 1 may be conservative, but nevertheless, provides technical simplicity for examining "objectivity" or "subjectivity" directly with respect to the existence of free parameters. In some situations, Definition 1 can be relaxed by including free parameters, but they all have to be determined solely from the given dataset.

Definition 2. Datasets in classification evaluations with a reject option. A reject option is sometimes considered for classifications in which one may assign samples to a reject or unknown class. Evaluations of classification with a reject option apply two datasets, namely, the output (or prediction) dataset $\left\{y_{k}\right\}_{k=1}^{n}$, which is a realization of discrete random variable $Y$ valued on set $\{1,2, \ldots, m+1\}$; and the target dataset $\left\{t_{k}\right\}_{k=1}^{n} \in T$ valued on set $\{1,2, \ldots, m\}$; where $n$ is the total number of samples, and $m$ is the total number of classes. A sample identified as a reject class is represented by $y_{k}=m+1$.

Remark 2. The term "abstaining classifiers" has been widely used in classification problems with a reject option [12][4]. However, most studies of abstaining classifications required cost matrices for their evaluations. The definition given above exhibits more generic scenarios in classification evaluations, because it does not require knowledge of cost terms for error types and reject types.

Definition 3. Augmented confusion matrix and its constraints [11]. An augmented confusion matrix includes one column for the reject class, which is added to a conventional confusion matrix:

$$
\mathbf{C}=\left[\begin{array}{ccccc}
c_{11} & c_{12} & \ldots & c_{1 m} & c_{1(m+1)} \\
c_{21} & c_{22} & \ldots & c_{2 m} & c_{2(m+1)} \\
& & \ldots & & \\
c_{m 1} & c_{m 2} & \ldots & c_{m m} & c_{m(m+1)}
\end{array}\right]
$$

where $c_{i j}$ represents the sample number of the $i$ th class that is classified as the $j$ th class. The row data corresponds to the actual classes, while the column data corresponds to the predicted classes. The last column represents the reject class. The relations and constraints of an augmented confusion matrix are:

$$
C_{j}=\sum_{j=1}^{m+1} c_{i j}, \quad C_{i}>0, \quad c_{i j} \geq 0, \quad i=1,2, \ldots, m,
$$


where $C_{i}$ is the total number for the $i$ th class, which is generally known in classification problems.

Definition 4. Error types and reject types. Following the conventions in binary classifications [? ], we denote $c_{12}$ and $c_{21}$ by "Type I Error" and "Type II Error" respectively; $c_{13}$ and $c_{23}$ by "Type I Reject" and "Type II Reject" respectively.

Definition 5. Normalized information measure. A normalized information measure, denoted as $\operatorname{NI}(T, Y) \in$ $[0,1]$, is a function based on information theory, which represents the degree of similarity between two random variables $T$ and $Y$.

In principle, we hope that all NI measures satisfy the three important properties, or axioms, of metrics [15][35], supposing $Z$ is another random variable:

$$
\begin{aligned}
& \text { P1: } N I(T, Y)=1 \text { iff } T=Y \text { (the identity axiom) } \\
& \text { P2: } N I(T, Y)+N I(Y, Z) \geq N I(T, Z) \text { (the triangle inequality) } \\
& \text { P3: } N I(T, Y)=N I(Y, T) \text { (the symmetry axiom) }
\end{aligned}
$$

Remark 3. Violations of properties of metrics are possible in reaching reasonable evaluations of classifications. For example, the triangle inequality and symmetry properties can be relaxed without changing the ranking orders among classifications if their evaluation measures are applied consistently. However, the identity property is indicated only for the relation $T=Y$ (assuming $T$ is padded with zeros to make it the same size as $Y$ ), and does not guarantee an exact solution $\left(t_{k}=y_{k}\right)$ in classifications (see Theorems 1 and 4 given later). If a violation of metric properties occurs, the NIs are referred to as measures, rather than metrics.

For classification evaluations, we consider the generic properties of metrics not to be as crucial in comparisons as certain specific features. In this work, we focus on specific features that, though not mathematically fundamental, are more necessary in classification applications. To select "better" measures for objective evaluations of classifications, we propose the following three desirable features together with their heuristic reasons.

Feature 1. Monotonicity with respect to the diagonal terms of the confusion matrix. The diagonal terms of the confusion matrix represent the exact classification numbers for all the samples. Or, they reflect the coincident numbers between $t$ and $y$ from a similarity viewpoint. When one of these terms changes, the evaluation measure should change in a monotonous way. Otherwise, any non-monotonic measure may fail to provide a rational result for ranking classifications correctly. This feature is originally proposed for describing the strength of agreement (or similarity) if the matrix is a contingency table [32].

Feature 2. Variation with reject rate. To improve classification performance, a reject option is often used in engineering applications [12]. Therefore, we suggest that a measure should be a scalar function on both classification accuracy and reject rates. Such a measure could be evaluated based solely on a given confusion matrix from a single operating point in the classification. This is different to the AUC measures that are based on an "Error-Reject" curve [16][24] from multiple operating points.

Feature 3. Intuitively consistent costs among error types and reject types. This feature is derived from the principle of our conventional intuitions when dealing with error types in classifications. It is also extended to reject types. Two specific intuitions are adopted for binary classifications. First, a misclassification or rejection from a small class will cause a greater cost than that from a large class. This intuition represents a property called "within error types and reject types". Second, a misclassification will produce a greater cost than a rejection from the same class, which is called "between error and reject types" property. If a measure is able to satisfy the intuitions, we refer to its associated costs as being "intuitively consistent". Exceptions may exist to the intuitions above, but we consider them as a very special case.

At this stage, it is worth discussing on "objectivity" in evaluations because one may doubt correctness of the intentions above and the terms "desirable" or "intuitions" in a study of objective evaluations. The three features seem to be "problematic" in terms of providing a general concept of "objectivity", because no human bias should be applied in the objective judgment of evaluation results. The following discussions justify the proposal of requiring desirable, or proper, features for objective measures. On one hand, we recognize that any evaluation will imply a certain degree of "subjectivity", since evaluations exist only as a result of human judgment. For examples, every selection of evaluation measures, even of objective ones, will rely on possible sources of "subjectivity" from users. On the other hand, engineering applications do concern about objective evaluations [29][32]. However, to the authors' best knowledge, 
a technical definition, or criterion, seems missing for determining objective or subjective measures in evaluations of classifications. For overcoming possible confusion and vagueness, we set Definition 1 as a practical criterion for examining whether a classification evaluation holds "objectivity" or does not. If a measure satisfies this definition, it will always retain the property of "objective consistency" in evaluating the given classification results. The three "desirable" features, though based on "intuitions" with "subjectivity", do not destroy the criterion of "objectivity" in classification evaluations. Therefore, it is logically correct to discuss "desirable" features of objective measures as long as the measures satisfy Definition 1 for keeping the defined "objectivity".

Note that all desirable features above are derived from our intuitions on general cases of classification evaluations. Other items may be derived for a wider examination of features. For example, Forbes [29] proposed six "constraints on proper comparative measures", namely, "statistically principled, readily interpretable, generalizable to $k$-class situations, not different to the special status, reflective of agreement, and insensitive to the segmentation". However, we consider the three features proposed in this work to be more crucial, especially as Feature 3 has never been concerned in previous studies of classification evaluations. Although Features 2 and 3 may share a similar meaning, they are presented individually to highlight their specific concerns. We can also call the desirable features "meta-measures", since these are defined to be qualitative and high-level measures about measures. In this work, we apply meta-measures in our investigation of information measures. The examination with respect to the meta-measures enables clarification of the causes of performance differences among the examined measures in classification evaluations. It will be helpful for users to understand advantages and limitations of different measures, either objective- or subjective-ones, from a higher level of evaluation knowledge.

\section{Normalized Information Measures based on Mutual Information}

All NI measures applied in this work are divided into one of three groups, namely, mutual-information based, divergence based, and cross-entropy based groups. In this section, we focus on the first group. Each measure in this group is derived directly from mutual information representing the degree of similarity between two random variables. For the purpose of objective evaluations, as suggested by Definition 1 in the previous section, we eliminate all candidate measures defined from the Renyi or Jensen entropies [36] [9] since they involve a free parameter. Therefore, without adding free parameters, we only apply the Shannon entropy to information measures [37]:

$$
H(Y)=-\sum_{y} p(y) \log _{2} p(y)
$$

where $Y$ is a discrete random variable with probability mass function $p(y)$. Then mutual information is defined as [37]:

$$
I(T, Y)=\sum_{t} \sum_{y} p(t, y) \log _{2} \frac{p(t, y)}{p(t) p(y)},
$$

where $p(t, y)$ is the joint distribution for the two discrete random variables $T$ and $Y$, and $p(t)$ and $p(y)$ are called marginal distributions that can be derived from:

$$
p(t)=\sum_{y} p(t, y), p(y)=\sum_{t} p(t, y)
$$

Sometimes, the simplified notations for $p_{i j}=p(t, y)=p\left(t=t_{i}, y=y_{j}\right)$ are used in this work. Table 1 lists the possible normalized information measures within the mutual-information based group. Basically, they all make use of Eq. (5) in their calculations. The main differences are due to the normalization schemes. In applying the formulas for calculating $N I_{k}$, one generally does not have an exact $p(t, y)$. For this reason, we adopt an empirical joint distribution defined below for the calculations.

Definition 6. Empirical joint distribution and empirical marginal distributions [11]. An empirical joint distribution is defined from the frequency means for the given confusion matrix, $\mathbf{C}$, as:

$$
P_{e}(t, y)=\left(P_{i j}\right)_{e}=\frac{1}{n} c_{i j}, \quad i=1,2, \ldots, m, \quad j=1,2, \ldots, m+1
$$


where $n=\sum C_{i}$, denotes the total number of samples in the classifications. The subscript " $e$ " is given for denoting empirical terms. The empirical marginal distributions are:

$$
\begin{gathered}
P_{e}\left(t=t_{i}\right)=\frac{C_{i}}{n}, \quad i=1,2, \ldots, m . \\
P_{e}\left(y=y_{j}\right)=\frac{1}{n} \sum_{i=1}^{m} c_{i j}, \quad j=1,2, \ldots, m+1 .
\end{gathered}
$$

Definition 7. Empirical mutual information [11]. The empirical mutual information is given by:

$$
I_{e}(T, Y)=\sum_{t} \sum_{y} P_{e}(t, y) \log _{2} \frac{P_{e}(t, y)}{P_{e}(t) P_{e}(y)}=\sum_{i=1}^{m} \sum_{j=1}^{m+1} \frac{c_{i j}}{n} \log _{2}\left(\frac{c_{i j}}{C_{i} \sum_{i=1}^{m} \frac{c_{i j}}{n}}\right) .
$$

Definitions 6 and 7 provide users with a direct means for applying information measures through the given data of the confusion matrix. For the sake of simplicity of analysis and discussion, we adopt the empirical distributions, or $p_{i j} \approx P_{i j}$, for calculating all NIs and deriving the theorems, but removing their associated subscript " $e$ ". Note that the notation of $\mathrm{NI}_{2}$ in Table 1 differs from the others for calculating mutual information, where $I_{M}(T, Y)$ is defined as "modified mutual information", The calculation of $I_{M}(T, Y)$ is carried out based on the intersection of T and Y. Hence, when using Eq. (8), the intersection requires that $I_{M}(T, Y)$ incorporate a summation of $j$ over 1 to $m$, instead of $m+1$. This definition is beyond mathematical rigor, but $N I_{2}$ has the same properties of metrics as $N I_{1}$. It was originally proposed to overcome the problem of unchanging values in NIs if rejections are made within only one class (referring to M9-M10 in Table 3, [11]). The following three theorems are derived for all NIs in this group.

Theorem 1. Within all NI measures in Table 1, when $N I(T, Y)=1$, the classification without a reject class may correspond to the case of either an exact classification $\left(y_{k}=t_{k}\right)$, or a specific misclassification $\left(y_{k} \neq t_{k}\right)$. The specific misclassification can be fully removed by simply exchanging labels in the confusion matrix.

Proof. If $N I(T, Y)=1$, we can obtain the following conditions from Eq. (8) for classifications without a reject class:

$$
p_{i j}=p\left(t=t_{i}\right) \approx P_{e}\left(t=t_{i}\right)=\frac{C_{i}}{n} \quad \text { and } \quad p_{k j}=0, \quad i, j, k=1,2, \ldots, m, \quad k \neq i .
$$

These conditions describe the specific confusion matrix where only one non-zero term appears in each column (with the exception of the last $(m+1)$ th column). When $j=i, \mathbf{C}$ is a diagonal matrix for representing an exact classification $\left(y_{k}=t_{k}\right)$. Otherwise, a specific misclassification exists for which a diagonal matrix can be obtained by exchanging labels in the confusion matrix (referring to M11 in Table 4, [11]). $\diamond$

Remark 4. Theorem 1 describes that $\mathrm{NI}(\mathrm{T}, \mathrm{Y})=1$ presents a necessary, but not sufficient, condition of an exact classification.

Theorem 2. For abstaining classification problems, when $N I(T, Y)=0$, the classifier generally reflects a misclassification. One special case is that all samples are considered to be one of $m$ classes, or be a reject class.

Proof. For NIs defined in Table $1, N I(T, Y)=0$ iff $I(T, Y)=0$. According to information theory [37], the following conditions can hold based on the given marginal distributions (or the empirical ones if a confusion matrix is used):

$$
I(T, Y)=0, \quad \text { iff } \quad p(t, y)=p(t) p(y) .
$$

The conditional part in Eq. (10) can be rewritten in the form $p_{i j}=p\left(t=t_{i}\right) p\left(y=y_{j}\right)$. From the constraints in (3), $p\left(t=t_{i}\right)>0(i=1,2, \ldots, m)$ can be obtained. For classification solutions, there should exist at least one term for $p\left(y=y_{j}\right)>0(j=1,2, \ldots, m+1)$. Therefore, at least one non-zero term for $p_{i j}>0(i \neq j)$ must be obtained. This non-zero term corresponds to the off-diagonal term in the confusion matrix, which indicates that a misclassification has occurred. When all samples have been identified as one of the classes (referring to M2 in Table 4, [11]), NI = 0 should be obtained. $\diamond$

Remark 5. Eq. (10) gives the statistical reason for zero mutual information, that is, the two random variables are "statistically independent". Theorem 2 demonstrates an intrinsic reason for local minima in NIs. 
Theorem 3. The NI measures defined by the Shannon entropy generally do not exhibit a monotonic property with respect to the diagonal terms of a confusion matrix.

Proof. Based on [11], we arrive at simpler conditions for the local minima about $I(T, Y)$ for the given confusion matrix:

$$
\mathbf{C}=\left[\begin{array}{cccc}
\ldots & 0 & 0 & \ldots \\
0 & c_{i, i} & c_{i, i+1} & 0 \\
0 & c_{i+1, i} & c_{i+1, i+1} & 0 \\
\ldots & 0 & 0 & \ldots
\end{array}\right], \quad \text { if } \quad \frac{c_{i, i}}{c_{i+1, i}}=\frac{c_{i, i+1}}{c_{i+1, i+1}} .
$$

The local minima are obtained because the four given non-zero terms in Eq. (11) produce zero (or the minimum) contribution to $I(T, Y)$. Suppose a generic form is given for $N I(T, Y)=g(I(T, Y))$, where $g(\cdot)$ is a normalization function. From the chain rule of derivatives, it can be seen that the conditions do not change for reaching the local minima. $\diamond$

Remark 6. The non-monotonic property of the information measures implies that these measures may suffer from an intrinsic problem of local minima for classification rankings (referring to M19-M20 in Table 4, [11]). Or, according to Feature 1 of the meta-measures, a rational result for the classification evaluations may not be obtained due to the non-monotonic property of the measures. This shortcoming has not been theoretically derived in previous studies $([28][29][32])$.

\section{Normalized Information Measures based on Information Divergence}

In this section, we propose normalized information measures based on the definition of information divergence. In Table 2, we summarize the commonly-used divergence measures, which are denoted as $D_{k}(T, Y)$ and represents dissimilarity between the two random variables $T$ and $Y$. In Sections 5 and 6, we apply the following notations for defining marginal distributions:

$$
p_{t}(z)=p_{t}(t=z)=p(t), \quad \text { and } \quad p_{y}(z)=p_{y}(y=z)=p(y),
$$

where $z$ is a possible scalar value that $t$ or $y$ can take. For a consistent comparison with the previous normalized information measures, we adopt the following transformation on $D_{k}[31]$ :

$$
N I_{k}=\exp \left(-D_{k}\right)
$$

This transformation provides both inverse and normalization functionalities. It does not introduce any extra parameters, and presents a high degree of simplicity, as in derivation for examining the local minima. Two more theorems are derived by following a similar analysis to that in the previous section.

Theorem 4. For all NI measures in Table 2, when $N I(T, Y)=1$, the classifier corresponds to the case of either an exact classification, or a specific misclassification. Generally, the misclassification in the latter case can not be removed by switching labels in the confusion matrix.

Proof. When $p_{y}(z)=p_{t}(z)$, it is always the case that $N I(T, Y)=1$. However, general conditions can be given for $p_{y}(z)=p_{t}(z)$ as follows:

$$
p_{y}\left(y=z_{i}\right)=p_{t}\left(t=z_{i}\right), \quad \text { or } \quad \sum_{j} p_{j i}=\sum_{j} p_{i j}, \quad i=1,2, \ldots, m .
$$

Eq. (14) implies two cases of classifications for $D_{k}(T, Y)=0, k=10, \ldots, 20$, One of these corresponds to an exact classification (or $y_{k}=t_{k}$ ), while the other is the result of a specific misclassification that shows the relationship of $y_{k} \neq t_{k}$, but $p_{y}(z)=p_{t}(z)$. In the latter case, switching labels in the confusion matrix to remove misclassification generally destroys the relation for $p_{y}(z)=p_{t}(z)$ at the same time. Considering the relation is a necessary condition for a perfect classification, the misclassification cannot be removed through a label switching operation. $\diamond$

Remark 7. Theorem 4 suggests the caution should be applied in explaining the classification evaluations when $N I(T, Y)=1$. The maximum of the NIs from the information divergence measures only indicates the equivalence between the marginal probabilities, $p_{y}(z)=p_{t}(z)$, but this is not always true for representing exact classifications (or 
$y_{k}=t_{k}$ ). Theorem 4 reveals an intrinsic problem when using an NI as a measure for similarity evaluations between two datasets, such as in image registration.

Theorem 5. The NI measures based on information divergence generally do not exhibit a monotonic property with respect to the diagonal terms of confusion matrix.

Proof. The theorem can be proved by examining the existence of multiple maxima for NI measures based on information divergence. Here we use a binary classification as an example. The local minima of $D_{k}$ are obtained when the following conditions exist for a confusion matrix:

$$
\mathbf{C}=\left[\begin{array}{ccc}
C_{1}-d_{1} & d_{1} & 0 \\
d_{2} & C_{2}-d_{2} & 0
\end{array}\right] \quad \text { and } \quad d_{1}=d_{2}
$$

where $d_{1}$ and $d_{2}$ are integer numbers (>0) for misclassified samples. The confusion matrix in Eq. (15) produces zero divergence $D_{k}$ and therefore, $N I_{k}=1$. However, changing from $d_{1} \neq d_{2}$ always results in $N I_{k}<1$. $\diamond$

Remark 8. Theorem 5 indicates another shortcoming of NIs in the information divergence group from the viewpoint of monotonicity. The reason is once again attributed to the usage of marginal distributions in calculations of divergence. The shortcoming has not been reported in previous investigations ([31] [35]).

\section{Normalized Information Measures based on Cross-Entropy}

In this section, we propose normalized information measures based on cross-entropy, which is defined for discrete random variables as [10]:

$$
H(T ; Y)=-\sum_{z} p_{t}(z) \log _{2} p_{y}(z), \quad \text { or } \quad H(Y ; T)=-\sum_{z} p_{y}(z) \log _{2} p_{t}(z) .
$$

Note that $H(T ; Y)$ differs from joint-entropy $H(T, Y)$ with respect to both notation and definition, and is given as [37]:

$$
H(T, Y)=-\sum_{t} \sum_{y} p(t, y) \log _{2} p(t, y)
$$

In fact, from Eq. (16), one can derive the relation between KL divergence (see Table 2) and cross-entropy:

$$
H(T ; Y)=H(T)+K L(T, Y), \quad \text { or } \quad H(Y ; T)=H(Y)+K L(Y, T) .
$$

If $H(T)$ is considered as a constant in classification since the target dataset is generally known and fixed, we can observe from Eq. (18) that cross-entropy shares a similar meaning as KL divergence for representing dissimilarity between $T$ and $Y$. From the conditions $H \geq 0$ and $K L \geq 0$, we are able to realize the normalization for cross-entropy shown in Table 3. Following similar discussions as in the previous section, we can derive that all information measures listed in Table 3 will also satisfy Theorems 4 and 5.

\section{Numerical Examples and Discussions}

This section presents several numerical examples together with associated discussions. All calculations for the numerical examples were done using the open source software Scilab 1 and a specific toolbox 2 . The detailed implementation of this toolbox is described in [38]. Table 4 lists six numerical examples in binary classification problems according to the specific scenarios of their confusion matrices. We adopt the notations from [39] for the terms "correct recognition rate $(C R)$ ", "error rate $(E)$ ", and "reject rate (Rej)" and their relation:

$$
C R+E+R e j=1
$$

In addition, we define "accuracy rate $(A)$ " as

\footnotetext{
${ }^{1}$ http://www.scilab.org

${ }^{2}$ The toolbox is freely available as the file "confmatrix 2 ni.zip" at (http://www.openpr.org.cn).
} 


$$
A=\frac{C R}{C R+E} .
$$

The first four classifications (or models) M1-M4 are provided to show the specific differences with respect to error types and reject types. In this work, we do not concern classifiers applied (say, neural networks or support vector machines) for evaluations, but only the resulting evaluations from these classifiers. In real applications, it is common to encounter ranking classification results as in M1 to M4. The first two classifications of M1 and M2 share the same values for the correct recognition and accuracy rates $(C R=A=99 \%)$. The other two classifications, for M3 and M4, have the same reject rates $(R e j=1 \%)$ and correct recognition rates $(C R=99 \%)$. The accuracy rates for M3 and M4 are also the same $(A=100 \%)$. This definition is consistent with the conventions in the study of "AccuracyReject" curves [16]. If neglecting the specific application backgrounds, users generally have a ranking order for the four classifications so that the "best" one is selected. The data from other conventional measures, such as Precision, Recall and $F 1$, are also given in Table 4. Without using extra knowledge about the cost of different error types or reject types, the conventional performance measures are not possible to rank the four classifications, M1-M4, properly.

According to the intuitions of Feature 3 proposed in Section 3, one can gain two sets of ranking orders for the four classifications M1 to M4 in forms of:

$$
\begin{array}{ll}
\mathfrak{R}(M 2)>\mathfrak{R}(M 1), & \mathfrak{R}(M 4)>\mathfrak{R}(M 3), \\
\mathfrak{R}(M 4)>\mathfrak{R}(M 2), & \mathfrak{R}(M 3)>\mathfrak{R}(M 1),
\end{array}
$$

where we denote $\mathfrak{R}(\bullet)$ to be a ranking operator, so that $\mathfrak{R}\left(M_{i}\right)>\mathfrak{R}\left(M_{j}\right)$ expresses $M_{i}$ is better than $M_{j}$ in ranking. From eq. (21), one is unable to tell the ranking order between M2 and M3. For a fast comparison, a specific letter is assigned to the ranking order of each model in Table 4 based on eq. (21):

$$
\mathfrak{R}(M 4)=A, \mathfrak{R}(M 3)=B, \mathfrak{R}(M 2)=B, \mathfrak{R}(M 1)=C .
$$

The top rank "A" indicates the "best" classification (M4 in this case) of the four models. Table 4 does not distinguish ranking order between M2 and M3. However, numerical investigations using information measures will provide the ranking order from the given data. The other two models, M5 and M6, are also specifically designed for the purpose of examining information measures on Theorems 3 and 5 (or Feature 1), respectively.

Tables 5 and 6 present the results on information measures for M1-M6, where the ranking orders among M1-M4 is based on the calculation results of NIs with the given digits. The following observations are achieved from the solutions to the examples.

1) When normalization functions include the term $H(Y)$ for the mutual information group, the associated NI produces the desirable feature of a variation in reject rate. $N I_{2}$ is effective for this feature even if it only uses $H(T)$ for its normalization. The effectiveness is attributed to the definition of $I_{M}(T, Y)$ for calculating mutual information based on the intersection of $T$ and $Y$.

2) The results of M5 and M6 confirm, respectively, Theorem 3 for local minima and Theorem 5 for maxima of NIs. The existence of multi extrema indicates the non-monotonic property with respect to the diagonal terms of the confusion matrix, thereby exhibiting an intrinsic shortcoming of the information measures.

3) For classifications M1 to M4, the meta-measure of Feature 3 suggests ranking orders as shown in eqs. (21) or (22). However, of all the measures in the three groups only $N I_{2}$ shows any consistency with the intuitions from the given examples (Tables 5 and 6). This result indicates that Feature 3 seems to be a difficult property for most information measures.

4) None of the performance or information measures investigated in this work fully satisfy the meta-measures. Examining data distinguishability in M1 through M4, we consider the information measures from the mutualinformation group to be more appropriate than those of the other groups (say, $N I_{12}$ and $N I_{22}$ do not show significant distinguishability, or value differences, to the four models). 
The fourth observation supports the proposal of meta-measures for a higher level of classification evaluations. The meta-measures provide users with a simple guideline of selecting "proper" measures from their specific concerns of applications. For example, the performance measures (such as $A, E, C R, F 1$, or AUC) satisfy Feature 1, but fail directly to distinguish error types and reject types in an objective evaluation. When Feature 2 or 3 is a main concern, the information measures exhibited to be more effective, despite them not being perfect.

Of all the information measures investigated, $\mathrm{NI}_{2}$ is shown to be the "best" for the given examples in terms of Feature 3. Therefore, more detailed studies, from both theoretical and numerical ones, were made on this promising measure. The theoretical properties of this measure was derived in Appendix A. While Theorem A1 confirms that $\mathrm{NI}_{2}$ satisfy Feature 3 around the exact classifications, Theorem A2 indicates that this measure is able to adjust the ranking order between a misclassification of a large class and a rejection of a small class. Table 7 shows two sets of confusions matrices which are similar to M1-M4 in Table 4. One can observe the changes of ranking orders among them. These changes numerically confirm Theorem A2 and its critical point, or cross-over point $\left(\Omega=C_{1} / n \approx 0.942\right)$, for the given data.

Further investigations were carried out on three-class problems. Although some NIs could be removed directly based on their poor performance with respect to the meta-measures (such as $N I_{1}$ and $N I_{9}$ on Feature 2), they were retained to demonstrate pros and cons in the applications. At this stage, we extend the concepts of error types and reject types to multiple classes. Nine examples are specifically designed in Table 8 . The ranking order for each model is shown in Table 8, which is derived from the intuitions of Feature 3. From Tables 9 and 10, it is interesting to see that $N I_{2}$ is still the most appropriate measure for classification evaluations. Using this measure, we can select the "best" and "worst" classifications consistent with our intuition. All other measures perform below our satisfactions for distinguishing error types and reject types properly.

The numerical study supports the viewpoint that no universally superior measure exists. For example, in comparing with information measure $\mathrm{NI}_{2}$, the conventional accuracy measure satisfies Feature 1, but does not qualify to Feature 3. Thus, any measure, either performance-based or information-based, should be designed and evaluated within the context of the specific applications. It is evident that the desirable features in the specific applications become more crucial (or "proper") for evaluation measures than some generic mathematical properties. For example, information measures (such as KL divergence), that may not satisfy a metric's properties (say, symmetry), are able to process classification evaluations including a reject option. They provide more applicable power than the conventional performance measures in abstaining classifications. However, we still need a complete picture about information measures with respect to their advantages as well as limitations. The examples in Tables 4, 7, and 8 only present limited scenarios for variations in confusion matrices. Using the open-source toolbox from [38], one is able to test more scenarios for numerical investigations.

\section{Summary}

In this work, we investigated objective evaluations of classifications by introducing normalized information measures. We reviewed the related works and discussed objectivity and its formal definition in evaluations. Objective evaluations may be required under different application background. In classifications, for example, exact knowledge of misclassification costs is sometimes unknown in evaluations. Moreover, cases of ignorance regarding reject costs appear more often in scenarios of abstaining classifications. In these cases, although subjective evaluations can be applied, the user-given data of the unknown abstention costs will lead to a much higher degree of uncertainty or inconsistency. We believe that an objective evaluation can be a suitable solution, as well as a complementary, approach to subjective evaluations. In some situations, an objective evaluation is considered useful despite the subjective evaluations being reasonable for the applications. The results from both objective and subjective evaluations give users an overall quality of classification results.

Considering that abstaining classifications are becoming more popular, we focused on the distinctions of error types and reject types within objective evaluations of classifications. First, we proposed three meta-measures for assessing classifications, which seem more relevant and proper than the properties of metrics in the context of classification applications. The meta-measures provide users with useful guidelines for a quick selection of candidate measures. Second, we tried systematically to enrich a classification evaluation bank by including commonly used information measures. Contrary to the conventional performance measures that apply empirical formulas, the information measures are theoretically more sound for objective evaluations of classifications. The key advantage of these 
measures over the conventional ones is their ability to handle multi-class classification evaluations with a reject option. Third, we revealed theoretically the intrinsic shortcomings of the information measures. These have not been formally reported before in studies of image registration, feature selection, or similarity ranking. The discovery of these shortcomings is very important for users to interpret their results correctly when applying those measures.

Based on the principle of the "No Free-Lunch Theorem" [15], we recognize that there are no "universally superior" measures [5]. It is not our aim to replace the conventional performance measures, but to explore information measures systematically in classification evaluations. The theoretical study demonstrates the strength and weakness of the information measures. Numerical investigations, conducted on binary and three-class classifications, confirmed that objective evaluations are not an easy topic in the study of machine learning. One of the most challenging tasks will be an exploration of novel measures that satisfy all meta-measures as well as the metric properties in objective evaluations of classifications. It is also necessary to define the "ranking order" intuitions among error types and reject types in generic classifications, which will form the basis of the quantitative meta-measures. However, this task becomes more difficult if classifications are beyond two classes.

\section{Acknowledgment}

This work is supported in part by Natural Science of Foundation of China (\#61075051).

\section{Appendix A. Theorems and Sensitivity Functions of $\mathrm{NI}_{2}$ for Binary Classifications}

Theorem A1: For a binary classification defined by:

$$
\begin{gathered}
\mathbf{C}=\left[\begin{array}{ccc}
T N & F P & R N \\
F N & T P & R P
\end{array}\right], \quad \text { and } \\
\mathrm{C}_{1}=T N+F P+R N, C_{2}=F N+T P+R P, C_{1}+C_{2}=n
\end{gathered}
$$

$\mathrm{NI}_{2}$ satisfies Feature 3 on the property regarding error types and reject types around the exact classifications. Specifically for the four confusion matrices below:

$$
M_{1}=\left[\begin{array}{ccc}
C_{1} & 0 & 0 \\
d & C_{2}-d & 0 \\
C_{1} & 0 & 0 \\
0 & C_{2}-d & d
\end{array}\right], \quad M_{2}=\left[\begin{array}{ccc}
C_{1}-d & d & 0 \\
0 & C_{2} & 0 \\
C_{1}-d & 0 & d \\
0 & C_{2} & 0
\end{array}\right],
$$

the following relations will be held:

$$
\begin{gathered}
N I_{2}\left(M_{1}\right)<N I_{2}\left(M_{2}\right) \quad \text { and } \quad N_{2}\left(M_{3}\right)<N I_{2}\left(M_{4}\right) \\
N I_{2}\left(M_{1}\right)<N I_{2}\left(M_{3}\right) \quad \text { and } \quad N I_{2}\left(M_{2}\right)<N I_{2}\left(M_{4}\right), \\
\text { where } \quad C_{1}>C_{2}>d>0 .
\end{gathered}
$$

Proof. For a binary classification, $\mathrm{NI}_{2}$ is defined by the modified mutual information:

$$
\begin{aligned}
& N_{2}=\frac{I_{M}(T, Y)}{H(T)}, \quad \text { and } \\
& \quad \begin{array}{l}
I_{M}(T, Y)=\frac{T N}{n} \log _{2} \frac{n T N}{C_{1}(T N+F N)}+\frac{F P}{n} \log _{2} \frac{n F P}{C_{1}(T P+F P)} \\
\quad+\frac{F N}{n} \log _{2} \frac{n F N}{C_{2}(F N+T N)}+\frac{T P}{n} \log _{2} \frac{n T P}{C_{2}(F P+T P)} .
\end{array}
\end{aligned}
$$

Let $M_{0}$ be a confusion matrix corresponding to the exact classifications:

$$
M_{0}=\left[\begin{array}{ccc}
C_{1} & 0 & 0 \\
0 & C_{2} & 0
\end{array}\right] .
$$


Based on the definition of $I_{M}$ in (A4), one can calculate the mutual information differences between two models. Considering $M_{0}$ to be a baseline, we obtain the analytical results below for the four models:

$$
\begin{gathered}
\Delta I_{10}=I_{M}\left(M_{1}\right)-I_{M}\left(M_{0}\right)=\frac{1}{n}\left(C_{1} \log _{2} \frac{C_{1}}{C_{1}+d}+d \log _{2} \frac{d}{C_{1}+d}\right), \\
\Delta I_{20}=I_{M}\left(M_{2}\right)-I_{M}\left(M_{0}\right)=\frac{1}{n}\left(C_{2} \log _{2} \frac{C_{2}}{C_{2}+d}+d \log _{2} \frac{d}{C_{2}+d}\right), \\
\Delta I_{30}=I_{M}\left(M_{3}\right)-I_{M}\left(M_{0}\right)=\frac{d}{n}\left(\log _{2} \frac{C_{2}}{n}\right), \\
\Delta I_{40}=I_{M}\left(M_{4}\right)-I_{M}\left(M_{0}\right)=\frac{d}{n}\left(\log _{2} \frac{C_{1}}{n}\right),
\end{gathered}
$$

For the given assumption $C_{1}>C_{2}>d>0$, all $\Delta I$ s above are negative values so that their abstracts represent the absolute costs in classifications. One can directly prove that $\left|\Delta I_{30}\right|>\left|\Delta I_{40}\right|$ from (A6-c) and (A6-d). The procedures for the proof of $\left|\Delta I_{10}\right|>\left|\Delta I_{20}\right|$ are given below. First, one needs to confirm the following two functions to be strictly decreasing $\left(x_{1}<x_{2}, g\left(x_{1}\right)>g\left(x_{2}\right)\right)$ :

$$
g_{1}(x)=\left(\frac{x}{x+d}\right)^{x} \quad \text { and } \quad g_{2}(x)=\left(\frac{d}{x+d}\right)^{d} \quad \text { for } \quad x>0, d>0 .
$$

Then, from the monotonically decreasing property of (A7-a), one can derive the following relations:

$$
\begin{aligned}
& C_{1}>C_{2} \rightarrow\left(\frac{C_{1}}{C_{1}+d}\right)^{C_{1}}<\left(\frac{C_{2}}{C_{2}+d}\right)^{C_{2}}<1 \text { and }\left(\frac{d}{C_{1}+d}\right)^{d}<\left(\frac{d}{C_{2}+d}\right)^{d}<1 \\
& \rightarrow \frac{1}{n}\left|C_{2} \log _{2} \frac{C_{2}}{C_{2}+d}+d \log _{2} \frac{d}{C_{2}+d}\right|<\frac{1}{n}\left|C_{1} \log _{2} \frac{C_{1}}{C_{1}+d}+d \log _{2} \frac{d}{C_{1}+d}\right| \\
& \rightarrow\left|\Delta I_{20}\right|<\left|\Delta I_{10}\right|
\end{aligned}
$$

The relations in (A3-a) are achieved for $N I_{2}$ because its normalization term, $H(T)$, is a constant for the given $C_{1}$ and $C_{2}$. One therefore confirms the satisfaction of Feature 3 on the property of the within error types and reject types around the exact classifications, respectively.

Then it is a proof of the relation (A3-b), which suggests that a misclassification suffer a higher cost than a rejection for the same class. Feature 3 considers this relation as a basic property in classifications for the between error and reject types. The procedures for the proof are:

$$
\begin{aligned}
& C_{1}>C_{2} \rightarrow C_{1} C_{2}+C_{1} d>\left(C_{1}+C_{2}\right) d=n d \\
& \rightarrow 1>\frac{C_{1}}{n}>\frac{d}{C_{2}+d} \rightarrow\left|\log _{2}\left(\frac{C_{1}}{n}\right)\right|<\left|\log _{2}\left(\frac{d}{C_{2}+d}\right)\right| \\
& \rightarrow \frac{1}{n}\left|d \log _{2}\left(\frac{C_{1}}{n}\right)\right|<\frac{1}{n}\left|d \log _{2} \frac{d}{C_{2}+d}\right|<\frac{1}{n}\left|C_{2} \log _{2} \frac{C_{2}}{C_{2}+d}+d \log _{2} \frac{d}{C_{2}+d}\right| \\
& \rightarrow\left|\Delta I_{40}\right|<\left|\Delta I_{20}\right| \\
& C_{1}+d<n \rightarrow C_{1}\left(C_{1}+d\right)+n d<C_{1} n+n d \rightarrow \frac{C_{1}\left(C_{1}+d\right)+n d}{n\left(C_{1}+d\right)}<1 \\
& \rightarrow \frac{C_{1}}{n}+\frac{d}{C_{1}+d}<1 \rightarrow \frac{d}{C_{1}+d}<\frac{C_{2}}{n}<1 \rightarrow\left|\log _{2} \frac{C_{2}}{n}\right|<\left|\log _{2} \frac{d}{C_{1}+d}\right| \\
& \rightarrow \frac{1}{n}\left|d \log _{2} \frac{C_{2}}{n}\right|<\frac{1}{n}\left|C_{1} \log _{2} \frac{C_{1}}{C_{1}+d}+d \log _{2} \frac{d}{C_{1}+d}\right| \\
& \rightarrow\left|\Delta I_{30}\right|<\left|\Delta I_{10}\right| .
\end{aligned}
$$

$\diamond$

Theorem A2: For the given conditions (A1)-(A2) and $C_{1}>C_{2}>d>0, N I_{2}$ will satisfy the following relations:

$$
\begin{array}{lll}
N I_{2}\left(M_{4}\right)>N I_{2}\left(M_{3}\right)>N I_{2}\left(M_{2}\right)>N I_{2}\left(M_{1}\right) & \text { for } & 0.5<p_{1}<\Omega \leq 1 \\
N I_{2}\left(M_{4}\right)>N I_{2}\left(M_{2}\right)>N I_{2}\left(M_{3}\right)>N I_{2}\left(M_{1}\right) & \text { for } & 0.5<\Omega<p_{1} \leq 1
\end{array}
$$

where we set $p_{1}=C_{1} / n$, and $\Omega$ is an upper boundary for the validation of (A9-a).

Proof. The first relation describes that the ranking order in (A9-a) is valid only for a certain range of $p_{1}$. The lower boundary is resulted from the assumption of $C_{1}$ to be a large class. The upper boundary, $\Omega$, is determined by 


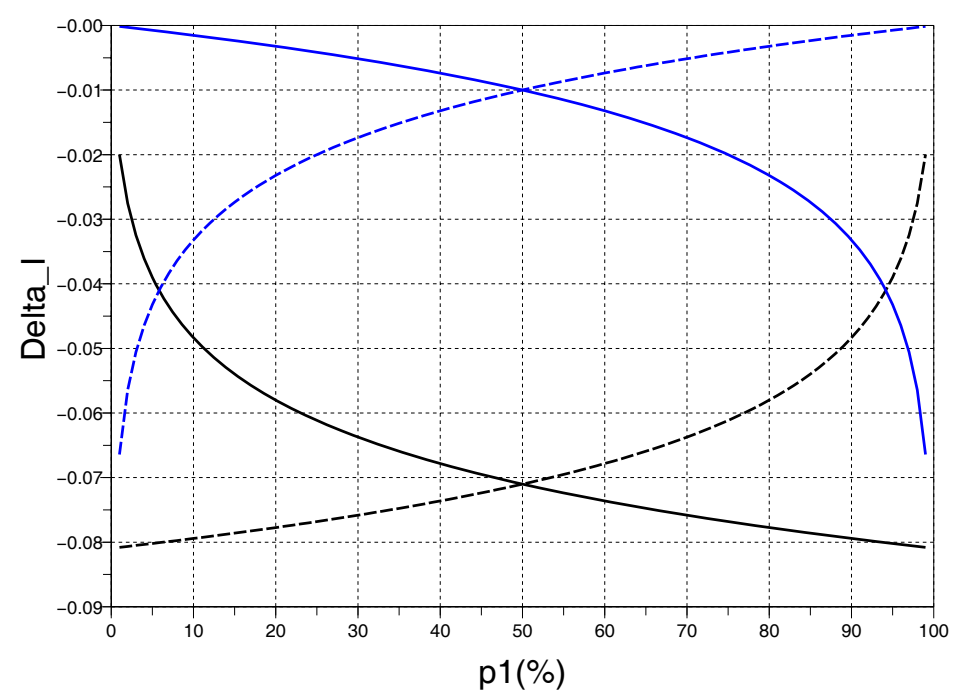

Figure A1.: Plots of " $\Delta I$ vs. $p_{1}(\%)$ " when $n=100$ and $d=1$.(Black-Solid $=\Delta I_{10}$, Black-Dash $=\Delta I_{20}$, Blue-Solid $=\Delta I_{30}$, Blue-Dash $\left.=\Delta I_{40}\right)$

the cross-over point between the functions of (A-6b) and (A-6c). For better understanding of the relations (A9), we present the plots of " $\Delta I$ vs. $p_{1}$ " when $n=100$ and $d=1$ (Fig. A1).

For examining the validation range of (A9-a), one needs to calculate the cross-over point from solving the equation below:

$$
f=\Delta I_{20}-\Delta I_{30}=\frac{1}{n}\left(C_{2} \log _{2} \frac{C_{2}}{C_{2}+d}+d \log _{2} \frac{d n}{C_{2}+d}\right)=0 .
$$

There exists no closed-form solution to $\Omega$. Based on the monotonicity of the related functions and relations in (A3), one is able to confirm the conditions in (A9-a) and (A9-b), respectively. Fig. A1 depicts numerically that only a single cross-over point appears to the range of $p_{1}>0.5\left(\right.$ or $\left.C_{1}>C_{2}\right)$. $\diamond$

Remark A1: We can denote $\Omega(n, d)$ to be the cross-over point obtained from $f$, with two independent variables $n$ and $d$. The value of $\Omega$ increases with $n$, but decreases with $d$. A numerical solution to $\Omega$ should be engaged. The physical interpretation of $\Omega$ is a critical point at which a rejection within a small class has the same cost with a misclassification within a large class. This situation generally does not occur except for classifications of largelyskewed classes (say, $C_{1}>>C_{2}$ ). Therefore, we call the ranking order in (A9-a) is a general ranking order, and one in (A9-b) is a largely-skewed-class ranking order.

Sensitivity functions: The sensitivity functions are given as the conventional forms for delivering approximation analysis of $I_{M}$ :

$$
\begin{gathered}
\frac{\partial I_{M}}{\partial T N}=\frac{1}{n}\left[\log _{2} \frac{n}{C_{1}}+\left(\log _{2} \frac{T N}{T N+F N}\right) \operatorname{sng}(T N)\right], \\
\frac{\partial I_{M}}{\partial T P}=\frac{1}{n}\left[\log _{2} \frac{n}{C_{2}}+\left(\log _{2} \frac{T P}{T P+F P}\right) \operatorname{sng}(T P)\right], \\
\frac{\partial I_{M}}{\partial F N}=\frac{1}{n}\left[\log _{2} \frac{n}{C_{2}}+\left(\log _{2} \frac{F N}{F N+T N}\right) \operatorname{sng}(F N)\right], \\
\frac{\partial I_{M}}{\partial F P}=\frac{1}{n}\left[\log _{2} \frac{n}{C_{1}}+\left(\log _{2} \frac{F P}{F P+T P}\right) \operatorname{sng}(F P)\right], \\
\frac{\partial I_{M}}{\partial R N}=-\frac{\partial I}{\partial T N}-\frac{\partial I}{\partial F P},
\end{gathered}
$$




$$
\frac{\partial I_{M}}{\partial R P}=-\frac{\partial I}{\partial F N}-\frac{\partial I}{\partial T P}
$$

where $\operatorname{sgn}($.$) is a sign function for satisfying the definition of H(0)=0$. Only four independent variables describe the sensitivity functions due to the two constraints in (A1-b). Hence, a chain rule is applied for deriving the functions of (A11-e) and (A11-f). $\diamond$

Remark A2: Using eq.(A11), we failed to reach the reasonable conclusions as those in Theorems A1 for the reason that the first-order differentials may be not sufficient for the analysis around the exact classifications. For example, we got the results for:

$$
\begin{aligned}
I\left(M_{1}\right) & -I\left(M_{0}\right) \approx\left(T P_{1}-T P_{0}\right) \frac{\partial I_{M}\left(M_{0}\right)}{\partial T P}+\left(F N_{1}-F N_{0}\right) \frac{\partial I_{M}\left(M_{0}\right)}{\partial F N} \\
& =-\frac{d}{n} \log _{2}\left(\frac{n}{C_{2}}\right)+\frac{d}{n} \log _{2}\left(\frac{n}{C_{2}}\right)=0 . \\
I\left(M_{2}\right) & -I\left(M_{0}\right) \approx\left(T N_{1}-T N_{0}\right) \frac{\partial I_{M}\left(M_{0}\right)}{\partial T N}+\left(F P_{1}-F P_{0}\right) \frac{\partial I_{M}\left(M_{0}\right)}{\partial F P} \\
& =-\frac{d}{n} \log _{2}\left(\frac{n}{C_{1}}\right)+\frac{d}{n} \log _{2}\left(\frac{n}{C_{1}}\right)=0 .
\end{aligned}
$$

This observation suggests that one needs to be cautious when using sensitivity function for approximation analysis on $I_{M}\left(\right.$ or $\left.\mathrm{NI}_{2}\right)$.

\section{References}

[1] C. X. Ling, J. Huang, H. Zhang, Auc: a statistically consistent and more discriminating measure than accuracy, in: the 18th International Conference on Artificial Intelligence (IJCAI-2003), 2003, pp. 519-526.

[2] M. M. S. Beg, A subjective measure of web search quality, Information Sciences 169 (2005) 365-381.

[3] N. Japkowicz, Why question machine learning evaluation methods?, in: AAAI-06 Evaluation Methods for Machine Learning Workshop, 2006, pp. 6-11.

[4] T. Pietraszek, Classification of intrusion detection alerts using abstaining classifiers, Intelligent Data Analysis 11 (2007) $293-316$.

[5] N. Lavesson, P. Davidsson, Analysis of multi criteria methods for algorithm and classifier evaluation, in: the 24th Annual Workshop of the Swedish, 2007.

[6] S. Vanderlooy, E. Hüllermeier, A critical analysis of variants of the auc, Machine Learning 72 (3) (2008) $247-262$.

[7] D. J. Hand, Measuring classifier performance: a coherent alternative to the area under the roc curve, Machine Learning 77 (1) (2009) $103-123$.

[8] Y. Y. Yao, S. K. M. Wong, C. J. Butz, On information theoretic measures of attribute importance, in: PAKDD, Beijing, China, 1999, pp. 133-137.

[9] J. Principe, D. Xu, Q. Zhao, J. Fisher, Learning from examples with information-theoretic criteria, J. VLSI Signal Proc 26 (2000) $61-77$.

[10] C. M. Bishop, Neural Networks for Pattern Recognition, Clarendon Press, London, 1995.

[11] B. G. Hu, Y. Wang, Evaluation criteria based on mutual information for classifications including rejected class, Acta Automatica Sinica 34 (2008) 1396-1403.

[12] M.-R. Temanni, S. A. Nadeem, D. Berrar, J. D. Zucker, Optimizing abstaining classifiers using roc analysis, in: CAMDA, 2007.

[13] P. Domingos, Metacost: A general method for making classifiers cost-sensitive, in: the 5th ACM SIGKDD International Conference on Knowledge Discovery and Data Mining, 1999, pp. 155-164.

[14] C. Elkan, Metacost: A general method for making classifiers cost-sensitive, in: the 17th International Joint Conference on Artificial Intelligence (IJCAI-01), 2001, pp. 973-978.

[15] R. O. Duda, P. E. Hart, D. Stork, Pattern Classification (2nd eds.), John Wiley, NY, 1995.

[16] D. J. C. Mackay, Information Theory, Inference, and Learning Algorithms, Cambridge: Cambridge University Press, 2003.

[17] C. Ferri, J. Hernández-Orallo, R. Modroiu, An experimental comparison of performance measures for classification, Pattern Recognition Letters 30 (2009) 27-38.

[18] N. Japkowicz, M. Shah, Evaluating Learning Algorithms: A Classification Perspective, Cambridge University Press, 2011.

[19] T. Fawcett, An introduction to roc analysis, Pattern Recognition Letters 27 (2006) 861-874.

[20] C. Drummond, R. C. Holte, Cost curves: An improved method for visualizing classifier performance, Machine Learning 65 (1) (2006) 95-130.

[21] A. Andersson, P. Davidsson, J. Linden, Measure-based classifier performance evaluation, Pattern Recognition Letters 20 (1999) $1165-1173$.

[22] C. de Stefano, C. Sansone, M. Vento, To reject or not to reject: That is the question - an answer in case of neural classifiers, IEEE Trans. on Systems, Man and Cybernetics 30 (2000) 84-94.

[23] T. Landgrebe, D. M. J. Tax, P. Paclik, R. P. W. Duin, The interaction between classification and reject performance for distance-based reject-option classifiers, Pattern Recognition Letters 27 (2006) 908-917.

[24] G. Iannello, G. Percannella, C. Sansone, P. Soda, On the use of classification reliability for improving performance of the one-per-class decomposition method, Data Knowl. Eng. 68 (2009) 1398-1410.

[25] S. Vanderlooy, I. Sprinkhuizen-Kuyper, E. Smirnov, J. van den Herik, The roc isometrics approach to construct reliable classifiers, Intelligent Data Analysis 13 (2009) 3-37.

[26] T. Kvålseth, Entropy and correlation: Some comments, IEEE Transactions on Systems, Man, and Cybernetics 17 (1987) 517-519.

[27] T. D. Wickens, Multiway Contingency Tables Analysis for the Social Sciences, Lawrence Erlbaum, Hillsdale, New Jersey, 1989. 
[28] J. T. Finn, Use of the average mutual information index in evaluating classification error and consistency, Inter. J. of Geographical Information Systems 7 (1993) 349-366

[29] A. Forbes, Classification-algorithm evaluation: Five performance measures based on confusion matrices, J. Clinical Monitoring and Computing 11 (1995) 189-206.

[30] I. Kononenko, I. Bratko, Information-based evaluation criterion for classifier's performance, Machine Learning 6 (1991) 67-80.

[31] R. Nishii, S. Tanaka, Accuracy and inaccuracy assessments in land-cover classification, IEEE Trans. Geoscience and Remote Sensing 37 (1999) 491-498.

[32] P.-N. Tan, V. Kumar, J. Srivastava, Selecting the right objective measure for association analysis, Information Systems 29 (2004) $293-313$.

[33] Y. Wang, B.-G. Hu, Derivations of normalized mutual information in binary classifications, in: the 6th International Conference on Fuzzy Systems and Knowledge Discovery, 2009, pp. 155-163.

[34] J. O. Berger, The case for objective bayesian analysis, Bayesian Analysis 1 (2005) 385-402.

[35] M. Li, X. Chen, X. Li, B. Ma, M. Vitanyi, The similarity metric, IEEE Trans. Information Theory 50 (2004) 3250-3264.

[36] C. Tsallis, Possible generalization of boltzmann-gibbs statistics, J. Stats. Physics 52 (1988) 479-487.

[37] T. M. Cover, J. A. Thomas, Elements of Information Theory, John Wiley, NY, 1995.

[38] B. G. Hu, Information measure toolbox for classifier evaluation on open source software scilab, in: IEEE International Workshop on Opensource Software for Scientific Computation, 2009, pp. 179-184.

[39] C. K. Chow, On optimum recognition error and reject tradeoff, IEEE Trans. on Information Theory 16 (1970) $41-46$.

[40] A. Strehl, J. Ghosh, Clustering ensembles, a knowledge reuse framework for combining multiple partitions, J. Machine Learning Research 3 (2002) 583-617.

[41] F. Malvestuto, Statistical treatment of the information content of a database, Information Systems 11 (1986) $211-223$.

[42] S. Kullback, R. A. Leibler, On information and sufficiency, The Annals of Mathematical Statistics 22 (1951) $79-86$.

[43] D. Johnson, S. Sinanovic, Symmetrizing the kullback-leibler distance, Rice University, Working Paper.

[44] I. Csiszar, Eine informationtheoretische ungleichung und ihre anwendung auf den beweis der ergodizitat von markoffschen ketten, Publ. Math. Inst. Hungar. Acad. Sci. 8 (1963) 85-108.

[45] J. Lin, Divergence measures based on the shannon entropy, IEEE Trans. Information Theory 37 (1991) $145-151$.

[46] D. Malerba, F. Esposito, M. Monopoli, Comparing dissimilarity measures for probabilistic symbolic objects, Data Mining III, Series Management Information Systems 6 (2002) 31-40. 
Table 1: NI measures within the mutual-information based group.

\begin{tabular}{lll}
\hline No. & Name [Reference] & Formula on $N I_{k}$ \\
\hline 1 & NI based on mutual information [28] & $N I_{1}(T, Y)=\frac{I(T, Y)}{H(T)}$ \\
2 & NI based on mutual information [11] & $N I_{2}(T, Y)=\frac{I_{M}(T, Y)}{H(T)}$ \\
3 & NI based on mutual information [28] & $N I_{3}(T, Y)=\frac{I(T, Y)}{H(Y)}$ \\
4 & NI based on mutual information & $\left.N I_{4}(T, Y)=\frac{1}{2} \prod_{\frac{I(T, Y)}{H(T)}}+\frac{I(T, Y)}{H(Y)}\right]$ \\
5 & NI based on mutual information [26] & $N I_{5}(T, Y)=\frac{2(T, Y)}{H(T)+H(Y)}$ \\
6 & NI based on mutual information [40] & $N I_{6}(T, Y)=\frac{I(T, Y)}{\sqrt{H(T) H(Y)}}$ \\
7 & NI based on mutual information [41] & $N I_{7}(T, Y)=\frac{I(T, Y)}{H(T, Y)}$ \\
8 & NI based on mutual information [26] & $N I_{8}(T, Y)=\frac{I(T, Y)}{\max (H(T), H(Y))}$ \\
9 & NI based on mutual information [26] & $N I_{9}(T, Y)=\frac{I(T, Y)}{\min (H(T), H(Y))}$ \\
\hline & &
\end{tabular}


Table 2: Information measures within the divergence based group.

\begin{tabular}{lll}
\hline No. & Name of $D_{k}$ [Reference] & Formula on $D_{k}\left(N I_{k}=\exp \left(-D_{k}\right)\right)$ \\
\hline 10 & ED-Quadratic Divergence [9] & $D_{10}=Q D_{E D}(T, Y)=\sum_{z}\left(p_{t}(z)-p_{y}(z)\right)^{2}$ \\
11 & CS-Quadratic Divergence [9] & $D_{11}=Q D_{C S}(T, Y)=\log _{2} \frac{\left.\sum_{t} p_{t}(z)^{2} \sum_{z} p_{y}(z)\right)^{2}}{\left[\sum_{z}\left(p_{t}(z) p_{y}(z)\right)^{2}\right.}$ \\
12 & KL Divergence [42] & $D_{12}=K L(T, Y)=\sum_{z} p_{t}(z) \log _{2} \frac{p_{t}(z)}{p_{y}(z)}$ \\
13 & Bhattacharyya Distance [43] & $D_{13}=D_{B}(T, Y)=-\log _{2} \sum_{z} \sqrt{p_{t}(z) p_{y}(z)}$ \\
14 & $\chi^{2}$ (Pearson) Divergence [44] & $D_{14}=\chi^{2}(T, Y)=\sum_{z} \frac{\left(p_{t}(z)-p_{y}(z)\right)^{2}}{p_{y}(z)}$ \\
15 & Hellinger Distance [44] & $D_{15}=H^{2}(T, Y)=\sum_{z}\left(\sqrt{p_{t}(z)}-\sqrt{\left.p_{y}(z)\right)^{2}}\right.$ \\
16 & Variation Distance [44] & $D_{16}=V(T, Y)=\sum_{z}\left|p_{t}(z)-p_{y}(z)\right|$ \\
17 & J divergence j [45] & $D_{17}=J(T, Y)=\sum_{z} p_{t}(z) \log _{2} \frac{p_{t}(z)}{p_{y}(z)}+\sum_{z} p_{y}(z) \log _{2} \frac{p_{y}(z)}{p_{1}(z)}$ \\
18 & L (or JS) divergence [45] & $D_{18}=L(T, Y)=K L(T, M)+K L(Y, M), M=\frac{\left(p_{t}(z)+p_{y}(z)\right)}{2}$ \\
19 & Symmetric $\chi^{2}$ Divergence [46] & $D_{19}=\chi_{S}^{2}(T, Y)=\sum_{z} \frac{\left(p_{t}(z)-p_{y}(z)\right)^{2}}{p_{y}(z)}+\sum_{z} \frac{\left(p_{y}(z)-p_{t}(z)\right)^{2}}{p_{t}(z)}$ \\
20 & Resistor Average Distance [43] & $D_{20}=D_{R A}(T, Y)=\frac{K L(T, Y) K L(Y, T)}{K L(T, Y)+K L(Y, T)}$ \\
\hline & &
\end{tabular}


Table 3: NI measures within the cross-entropy based group.

\begin{tabular}{lll}
\hline No. & Name & Formula on $N I_{k}$ \\
\hline 21 & NI based on cross-entropy & $N I_{21}=\frac{H(T)}{H(T ; Y)}, H(T ; Y)=-\sum_{z} p_{t}(z) \log _{2} p_{y}(z)$ \\
22 & NI based on cross-entropy & $N I_{22}=\frac{H(Y)}{H(Y ; T)}, H(Y ; T)=-\sum_{z} p_{y}(z) \log _{2} p_{t}(z)$ \\
23 & NI based on cross-entropy & $N I_{23}=\frac{1}{2}\left(\frac{H(T)}{H(T ; Y)}+\frac{H(Y)}{H(Y ; T)}\right)$ \\
24 & NI based on cross-entropy & $N I_{24}=\frac{H(T)+H(Y)}{H(T ; Y)+H(Y ; T)}$ \\
\hline
\end{tabular}

Table 4: Numerical examples in Binary Classifications(M1-M4 and M6: $C_{1}=90, C_{2}=10 ;$ M5: $C_{1}=95, C_{2}=5$ ). $(\mathrm{R})=$ ranking order for the model, where $\mathrm{R}=\mathrm{A}, \mathrm{B}, \ldots$, in descending order from the top.

\begin{tabular}{|c|c|c|c|c|c|c|c|c|c|c|c|c|c|c|c|c|c|c|}
\hline $\begin{array}{l}\text { Model } \\
\text { (Ranking) }\end{array}$ & $\begin{array}{l}\text { M1 } \\
\text { (C) }\end{array}$ & & & $\begin{array}{l}\text { M2 } \\
\text { (B) }\end{array}$ & & & $\begin{array}{l}\text { M3 } \\
\text { (B) }\end{array}$ & & & $\begin{array}{l}\text { M4 } \\
\text { (A) }\end{array}$ & & & M5 & & & M6 & & \\
\hline \multirow{2}{*}{ C } & 90 & 0 & 0 & 89 & 1 & 0 & 90 & 0 & 0 & 89 & 0 & 1 & 57 & 38 & 0 & 89 & 1 & 0 \\
\hline & 1 & 9 & 0 & 0 & 10 & 0 & 0 & 9 & 1 & 0 & 10 & 0 & 3 & 2 & 0 & 1 & 9 & 0 \\
\hline$C R$ & 0.990 & & & 0.990 & & & 0.990 & & & 0.990 & & & 0.590 & & & 0.980 & & \\
\hline$R e j$ & 0.000 & & & 0.000 & & & 0.010 & & & 0.010 & & & 0.000 & & & 0.000 & & \\
\hline Precision & 0.989 & & & 1.000 & & & 1.000 & & & 1.000 & & & 0.950 & & & 0.989 & & \\
\hline Recall & 1.000 & & & 0.989 & & & 1.000 & & & 1.000 & & & 0.600 & & & 0.989 & & \\
\hline$F 1$ & 0.994 & & & 0.994 & & & 1.000 & & & 1.000 & & & 0.735 & & & 0.989 & & \\
\hline
\end{tabular}


Table 5: Results for the models in Table 4 on information measures from mutual-information and cross-entropy groups. $(\mathrm{R})=$ ranking order for the model, where $\mathrm{R}=\mathrm{A}, \mathrm{B}, \ldots$, , in descending order from the top.

\begin{tabular}{|c|c|c|c|c|c|c|c|c|c|c|c|c|c|}
\hline Model & $N I_{1}$ & $\mathrm{NI}_{2}$ & $\mathrm{NI}_{3}$ & $\mathrm{NI}_{4}$ & $\mathrm{NI}_{5}$ & $N I_{6}$ & $\mathrm{NI}_{7}$ & $N I_{8}$ & $\mathrm{NI}_{9}$ & $N I_{22}$ & $\mathrm{NI}_{23}$ & $\mathrm{NI}_{24}$ & $\mathrm{NI}_{25}$ \\
\hline $\begin{array}{l}\text { M1 } \\
\text { (C) }\end{array}$ & $\begin{array}{l}0.831 \\
\text { (D) }\end{array}$ & $\begin{array}{l}0.831 \\
\text { (D) }\end{array}$ & $\begin{array}{l}0.893 \\
\text { (B) }\end{array}$ & $\begin{array}{l}0.862 \\
\text { (D) }\end{array}$ & $\begin{array}{l}0.860 \\
\text { (D) }\end{array}$ & $\begin{array}{l}0.861 \\
\text { (D) }\end{array}$ & $\begin{array}{l}0.755 \\
\text { (D) }\end{array}$ & $\begin{array}{l}0.831 \\
\text { (D) }\end{array}$ & $\begin{array}{l}0.893 \\
\text { (D) }\end{array}$ & $\begin{array}{l}0.998 \\
\text { (A) }\end{array}$ & $\begin{array}{l}0.998 \\
\text { (A) }\end{array}$ & $\begin{array}{l}0.998 \\
\text { (A) }\end{array}$ & $\begin{array}{l}0.998 \\
\text { (A) }\end{array}$ \\
\hline $\begin{array}{l}\text { M2 } \\
\text { (B) }\end{array}$ & $\begin{array}{l}0.897 \\
\text { (C) }\end{array}$ & $\begin{array}{l}0.897 \\
\text { (C) }\end{array}$ & $\begin{array}{l}0.841 \\
\text { (D) }\end{array}$ & $\begin{array}{l}0.869 \\
(\mathrm{C})\end{array}$ & $\begin{array}{l}0.868 \\
\text { (C) }\end{array}$ & $\begin{array}{l}0.869 \\
\text { (C) }\end{array}$ & $\begin{array}{l}0.767 \\
\text { (C) }\end{array}$ & $\begin{array}{l}0.841 \\
\text { (C) }\end{array}$ & $\begin{array}{l}0.897 \\
\text { (C) }\end{array}$ & $\begin{array}{l}0.998 \\
\text { (A) }\end{array}$ & $\begin{array}{l}0.998 \\
\text { (A) }\end{array}$ & $\begin{array}{l}0.998 \\
\text { (A) }\end{array}$ & $\begin{array}{l}0.998 \\
\text { (A) }\end{array}$ \\
\hline $\begin{array}{l}\text { M3 } \\
\text { (B) }\end{array}$ & $\begin{array}{l}1.000 \\
\text { (A) }\end{array}$ & $\begin{array}{l}0.929 \\
\text { (B) }\end{array}$ & $\begin{array}{l}0.909 \\
\text { (A) }\end{array}$ & $\begin{array}{l}0.955 \\
\text { (A) }\end{array}$ & $\begin{array}{l}0.952 \\
\text { (A) }\end{array}$ & $\begin{array}{l}0.953 \\
\text { (A) }\end{array}$ & $\begin{array}{l}0.909 \\
\text { (A) }\end{array}$ & $\begin{array}{l}0.909 \\
\text { (A) }\end{array}$ & $\begin{array}{l}1.000 \\
\text { (A) }\end{array}$ & $\begin{array}{l}0.969 \\
\text { (D) }\end{array}$ & $\begin{array}{l}0.000 \\
\text { (B) }\end{array}$ & $\begin{array}{l}0.484 \\
\text { (C) }\end{array}$ & $\begin{array}{l}0.000 \\
\text { (B) }\end{array}$ \\
\hline $\begin{array}{l}\text { M4 } \\
\text { (A) }\end{array}$ & $\begin{array}{l}1.000 \\
\text { (A) }\end{array}$ & $\begin{array}{l}0.997 \\
\text { (A) }\end{array}$ & $\begin{array}{l}0.855 \\
\text { (C) }\end{array}$ & $\begin{array}{l}0.928 \\
\text { (B) }\end{array}$ & $\begin{array}{l}0.922 \\
\text { (B) }\end{array}$ & $\begin{array}{l}0.925 \\
\text { (B) }\end{array}$ & $\begin{array}{l}0.855 \\
\text { (B) }\end{array}$ & $\begin{array}{l}0.855 \\
\text { (B) }\end{array}$ & $\begin{array}{l}1.000 \\
\text { (A) }\end{array}$ & $\begin{array}{l}0.970 \\
\text { (C) }\end{array}$ & $\begin{array}{l}0.000 \\
\text { (B) }\end{array}$ & $\begin{array}{l}0.485 \\
\text { (B) }\end{array}$ & $\begin{array}{l}0.000 \\
\text { (B) }\end{array}$ \\
\hline M5 & 0.000 & 0.000 & 0.000 & 0.000 & 0.000 & 0.000 & 0.000 & 0.000 & 0.000 & 0.374 & 0.548 & 0.461 & 0.495 \\
\hline M6 & 0.731 & 0.731 & 0.731 & 0.731 & 0.731 & 0.731 & 0.576 & 0.731 & 0.731 & 1.000 & 1.000 & 1.000 & 1.000 \\
\hline
\end{tabular}


Table 6: Results for the models in Table 4 on information measures from divergence group. $\mathrm{S}=$ singularity which cannot be removed. $(\mathrm{R})=$ ranking order for the model, where $\mathrm{R}=\mathrm{A}, \mathrm{B}, \ldots$,., in descending order from the top.

\begin{tabular}{llllllllllll} 
Model & $N I_{10}$ & $N I_{11}$ & $N I_{12}$ & $N I_{13}$ & $N I_{14}$ & $N I_{15}$ & $N I_{16}$ & $N I_{17}$ & $N I_{18}$ & $N I_{19}$ & $N I_{20}$ \\
\hline M1 & 0.9998 & 0.9998 & 0.9991 & 0.9998 & 0.9988 & 0.9997 & 0.9802 & 0.9983 & 0.9996 & 0.9977 & 0.9996 \\
$(\mathrm{C})$ & $(\mathrm{A})$ & $(\mathrm{A})$ & $(\mathrm{B})$ & $(\mathrm{A})$ & $(\mathrm{B})$ & $(\mathrm{A})$ & $(\mathrm{A})$ & $(\mathrm{B})$ & $(\mathrm{A})$ & $\begin{array}{l}\text { ( ) }) \\
(\mathrm{A})\end{array}$ \\
\hline M2 & 0.9998 & 0.9998 & 0.9992 & 0.9998 & 0.9990 & 0.9997 & 0.9802 & 0.9985 & 0.9996 & 0.9979 & 0.9996 \\
$(\mathrm{~B})$ & $(\mathrm{A})$ & $(\mathrm{A})$ & $(\mathrm{A})$ & $(\mathrm{A})$ & $(\mathrm{A})$ & $(\mathrm{A})$ & $(\mathrm{A})$ & $(\mathrm{A})$ & $(\mathrm{A})$ & $(\mathrm{A})$ & $(\mathrm{A})$ \\
\hline M3 & 0.9998 & 0.9996 & 0.9849 & 0.9926 & 0.9890 & 0.9898 & 0.9802 & $\mathrm{~S}$ & 0.9897 & $\mathrm{~S}$ & $\mathrm{~S}$ \\
(B) & $(\mathrm{A})$ & $(\mathrm{D})$ & $(\mathrm{D})$ & $(\mathrm{D})$ & $(\mathrm{D})$ & $(\mathrm{D})$ & $(\mathrm{A})$ & & $(\mathrm{D})$ & & \\
\hline M4 & 0.9998 & 0.9998 & 0.9856 & 0.9928 & 0.9899 & 0.9900 & 0.9802 & $\mathrm{~S}$ & 0.9900 & $\mathrm{~S}$ & $\mathrm{~S}$ \\
(A) & $(\mathrm{A})$ & $(\mathrm{A})$ & $(\mathrm{C})$ & $(\mathrm{C})$ & $(\mathrm{C})$ & $(\mathrm{C})$ & $(\mathrm{A})$ & & $(\mathrm{C})$ & & \\
\hline M5 & 0.7827 & 0.6473 & 0.6189 & 0.8540 & 0.6002 & 0.8129 & 0.4966 & 0.2775 & 0.7550 & 0.0455 & 0.7406 \\
\hline M6 & 1.0000 & 1.0000 & 1.0000 & 1.0000 & 1.0000 & 1.0000 & 1.0000 & 1.0000 & 1.0000 & 1.0000 & $\mathrm{~S}$ \\
\hline
\end{tabular}


Table 7: Numerical examples in Binary Classifications $(\mathrm{n}=100)$. $(\mathrm{R})=$ ranking order for the model, where $\mathrm{R}=\mathrm{A}, \mathrm{B}, \ldots$, in descending order from the top

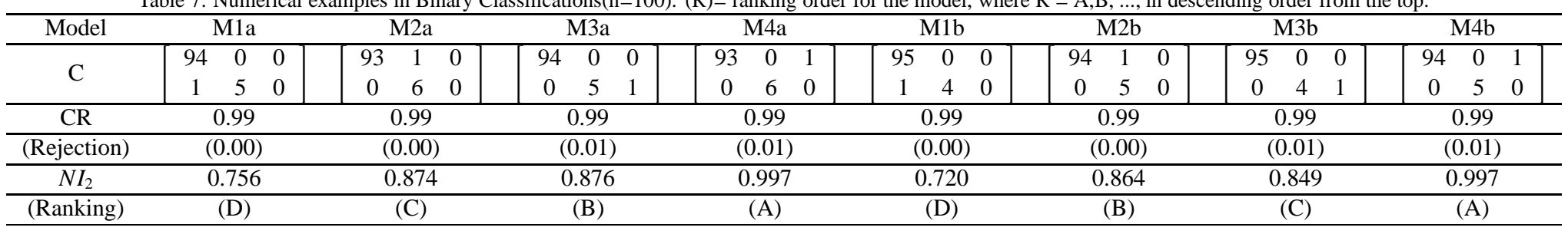


Table 8: Classification examples in three classes $\left(C_{1}=80, C_{2}=15, C_{3}=5\right) .(\mathrm{R})=$ ranking order for the model, where $\mathrm{R}=\mathrm{A}, \mathrm{B}, \ldots$, in descending order from the top.

\begin{tabular}{|c|c|c|c|c|c|c|c|c|c|c|c|c|c|c|c|c|c|c|c|c|}
\hline $\begin{array}{l}\text { Model } \\
\text { (Ranking) }\end{array}$ & $\begin{array}{l}\text { M7 } \\
\text { (C) }\end{array}$ & & & & $\begin{array}{l}\text { M8 } \\
\text { (C) }\end{array}$ & & & & $\begin{array}{l}\text { M9 } \\
\text { (B) }\end{array}$ & & & & $\begin{array}{l}\text { M10 } \\
\text { (B) }\end{array}$ & & & & $\begin{array}{l}\text { M11 } \\
\text { (B) }\end{array}$ & & & \\
\hline \multirow{3}{*}{ C } & 80 & 0 & 0 & 0 & 80 & 0 & 0 & 0 & 80 & 0 & 0 & 0 & 80 & 0 & 0 & 0 & 80 & 0 & 0 & 0 \\
\hline & 0 & 15 & 0 & 0 & 0 & 15 & 0 & 0 & 0 & 15 & 0 & 0 & 1 & 14 & 0 & 0 & 0 & 14 & 1 & 0 \\
\hline & 1 & 0 & 4 & 0 & 0 & 1 & 4 & 0 & 0 & 0 & 4 & 1 & 0 & 0 & 5 & 0 & 0 & 0 & 5 & 0 \\
\hline$C R$ & 0.99 & & & & 0.99 & & & & 0.99 & & & & 0.99 & & & & 0.99 & & & \\
\hline$R e j$ & 0.00 & & & & 0.00 & & & & 0.01 & & & & 0.00 & & & & 0.00 & & & \\
\hline Model & M12 & & & & M13 & & & & M14 & & & & M15 & & & & & & & \\
\hline (Ranking) & (B) & & & & (B) & & & & (B) & & & & (A) & & & & & & & \\
\hline \multirow{3}{*}{$\mathbf{C}$} & {$[80$} & 0 & 0 & 0 & {$[79$} & 1 & 0 & 0 & {$[79$} & 0 & 1 & 0 & {$[79$} & 0 & 0 & 1 & & & & \\
\hline & 0 & 14 & 0 & 1 & 0 & 15 & 0 & 0 & 0 & 15 & 0 & 0 & 0 & 15 & 0 & 0 & & & & \\
\hline & 0 & 0 & 5 & 0 & 0 & 0 & 5 & 0 & 0 & 0 & 5 & 0 & 0 & 0 & 5 & 0 & & & & \\
\hline$C R$ & 0.99 & & & & 0.99 & & & & 0.99 & & & & 0.99 & & & & & & & \\
\hline$R e j$ & 0.01 & & & & 0.00 & & & & 0.00 & & & & 0.01 & & & & & & & \\
\hline
\end{tabular}


Table 9: Results for the models in Table 8 on information measures from mutual-information and cross-entropy groups. $\mathrm{S}=$ singularity which cannot be removed. (R)= ranking order for the model, where $\mathrm{R}=\mathrm{A}, \mathrm{B}, \ldots$, in descending order from the top.

\begin{tabular}{|c|c|c|c|c|c|c|c|c|c|c|c|c|c|}
\hline Model & $N I_{1}$ & $\mathrm{NI}_{2}$ & $\mathrm{NI}_{3}$ & $\mathrm{NI}_{4}$ & $\mathrm{NI}_{5}$ & $N I_{6}$ & $\mathrm{NI}_{7}$ & $\mathrm{NI}_{8}$ & $\mathrm{NI}_{9}$ & $N I_{21}$ & $\mathrm{NI}_{22}$ & $\mathrm{NI}_{23}$ & $\mathrm{NI}_{24}$ \\
\hline $\begin{array}{l}\text { M7 } \\
(\mathrm{F})\end{array}$ & $\begin{array}{l}0.912 \\
(\mathrm{~F})\end{array}$ & $\begin{array}{l}0.912 \\
(\mathrm{~F})\end{array}$ & $\begin{array}{l}0.957 \\
\text { (C) }\end{array}$ & $\begin{array}{l}0.935 \\
(\mathrm{G})\end{array}$ & $\begin{array}{l}0.934 \\
(\mathrm{G})\end{array}$ & $\begin{array}{l}0.934 \\
(\mathrm{G})\end{array}$ & $\begin{array}{l}0.876 \\
(\mathrm{~F})\end{array}$ & $\begin{array}{l}0.912 \\
(\mathrm{H})\end{array}$ & $\begin{array}{l}0.957 \\
\text { (E) }\end{array}$ & $\begin{array}{l}0.998 \\
\text { (D) }\end{array}$ & $\begin{array}{l}0.998 \\
\text { (C) }\end{array}$ & $\begin{array}{l}0.998 \\
\text { (C) }\end{array}$ & $\begin{array}{l}0.998 \\
\text { (C) }\end{array}$ \\
\hline $\begin{array}{l}\text { M8 } \\
\text { (F) }\end{array}$ & $\begin{array}{l}0.939 \\
(\mathrm{E})\end{array}$ & $\begin{array}{l}0.939 \\
(\mathrm{E})\end{array}$ & $\begin{array}{l}0.958 \\
\text { (B) }\end{array}$ & $\begin{array}{l}0.949 \\
\text { (D) }\end{array}$ & $\begin{array}{l}0.949 \\
\text { (D) }\end{array}$ & $\begin{array}{l}0.949 \\
\text { (D) }\end{array}$ & $\begin{array}{l}0.902 \\
\text { (D) }\end{array}$ & $\begin{array}{l}0.939 \\
\text { (D) }\end{array}$ & $\begin{array}{l}0.958 \\
\text { (D) }\end{array}$ & $\begin{array}{l}0.998 \\
\text { (D) }\end{array}$ & $\begin{array}{l}0.998 \\
\text { (C) }\end{array}$ & $\begin{array}{l}0.998 \\
\text { (C) }\end{array}$ & $\begin{array}{l}0.998 \\
\text { (C) }\end{array}$ \\
\hline $\begin{array}{l}\text { M9 } \\
\text { (C) }\end{array}$ & $\begin{array}{l}1.000 \\
\text { (A) }\end{array}$ & $\begin{array}{l}0.951 \\
\text { (D) }\end{array}$ & $\begin{array}{l}0.961 \\
\text { (A) }\end{array}$ & $\begin{array}{l}0.980 \\
\text { (A) }\end{array}$ & $\begin{array}{l}0.980 \\
\text { (A) }\end{array}$ & $\begin{array}{l}0.980 \\
\text { (A) }\end{array}$ & $\begin{array}{l}0.961 \\
\text { (A) }\end{array}$ & $\begin{array}{l}0.961 \\
\text { (A) }\end{array}$ & $\begin{array}{l}1.000 \\
\text { (A) }\end{array}$ & $\begin{array}{l}0.982 \\
\text { (G) }\end{array}$ & $\begin{array}{l}0.000 \\
\text { (G) }\end{array}$ & $\begin{array}{l}0.491 \\
\text { (I) }\end{array}$ & $\begin{array}{l}0.000 \\
(\mathrm{G})\end{array}$ \\
\hline $\begin{array}{l}\text { M10 } \\
\text { (E) }\end{array}$ & $\begin{array}{l}0.912 \\
(\mathrm{~F})\end{array}$ & $\begin{array}{l}0.912 \\
(\mathrm{~F})\end{array}$ & $\begin{array}{l}0.938 \\
(\mathrm{~F})\end{array}$ & $\begin{array}{l}0.925 \\
\text { (I) }\end{array}$ & $\begin{array}{l}0.925 \\
\text { (I) }\end{array}$ & $\begin{array}{l}0.925 \\
\text { (I) }\end{array}$ & $\begin{array}{l}0.860 \\
(\mathrm{H})\end{array}$ & $\begin{array}{l}0.912 \\
(\mathrm{H})\end{array}$ & $\begin{array}{l}0.938 \\
(\mathrm{G})\end{array}$ & $\begin{array}{l}0.999 \\
\text { (A) }\end{array}$ & $\begin{array}{l}0.999 \\
\text { (A) }\end{array}$ & $\begin{array}{l}0.999 \\
\text { (A) }\end{array}$ & $\begin{array}{l}0.999 \\
\text { (A) }\end{array}$ \\
\hline $\begin{array}{l}\text { M11 } \\
\text { (E) }\end{array}$ & $\begin{array}{l}0.956 \\
\text { (D) } \\
\end{array}$ & $\begin{array}{l}0.956 \\
(\mathrm{C}) \\
\end{array}$ & $\begin{array}{l}0.941 \\
(\mathrm{E}) \\
\end{array}$ & $\begin{array}{l}0.948 \\
(\mathrm{E}) \\
\end{array}$ & $\begin{array}{l}0.948 \\
(\mathrm{E}) \\
\end{array}$ & $\begin{array}{l}0.948 \\
\text { (E) }\end{array}$ & $\begin{array}{l}0.902 \\
\text { (D) }\end{array}$ & $\begin{array}{l}0.941 \\
(\mathrm{C}) \\
\end{array}$ & $\begin{array}{l}0.956 \\
\text { (E) }\end{array}$ & $\begin{array}{l}0.998 \\
\text { (B) }\end{array}$ & $\begin{array}{l}0.998 \\
(\mathrm{C}) \\
\end{array}$ & $\begin{array}{l}0.998 \\
\text { (C) }\end{array}$ & $\begin{array}{l}0.998 \\
(\mathrm{C}) \\
\end{array}$ \\
\hline $\begin{array}{l}\text { M12 } \\
\text { (B) }\end{array}$ & $\begin{array}{l}1.000 \\
\text { (A) }\end{array}$ & $\begin{array}{l}0.969 \\
\text { (B) }\end{array}$ & $\begin{array}{l}0.943 \\
\text { (D) }\end{array}$ & $\begin{array}{l}0.972 \\
\text { (B) }\end{array}$ & $\begin{array}{l}0.971 \\
\text { (B) }\end{array}$ & $\begin{array}{l}0.971 \\
\text { (B) }\end{array}$ & $\begin{array}{l}0.943 \\
\text { (B) }\end{array}$ & $\begin{array}{l}0.943 \\
\text { (B) }\end{array}$ & $\begin{array}{l}1.000 \\
\text { (A) }\end{array}$ & $\begin{array}{l}0.983 \\
(\mathrm{~F})\end{array}$ & $\begin{array}{l}0.000 \\
\text { (G) }\end{array}$ & $\begin{array}{l}0.492 \\
\text { (G) }\end{array}$ & $\begin{array}{l}0.000 \\
(\mathrm{G})\end{array}$ \\
\hline $\begin{array}{l}\text { M13 } \\
\text { (D) }\end{array}$ & $\begin{array}{l}0.939 \\
(\mathrm{E})\end{array}$ & $\begin{array}{l}0.939 \\
(\mathrm{E})\end{array}$ & $\begin{array}{l}0.915 \\
\text { (I) }\end{array}$ & $\begin{array}{l}0.927 \\
(\mathrm{H})\end{array}$ & $\begin{array}{l}0.927 \\
(\mathrm{H})\end{array}$ & $\begin{array}{l}0.927 \\
(\mathrm{H})\end{array}$ & $\begin{array}{l}0.863 \\
(\mathrm{G})\end{array}$ & $\begin{array}{l}0.915 \\
\text { (G) }\end{array}$ & $\begin{array}{l}0.939 \\
(\mathrm{~F})\end{array}$ & $\begin{array}{l}0.999 \\
\text { (A) }\end{array}$ & $\begin{array}{l}0.999 \\
\text { (A) }\end{array}$ & $\begin{array}{l}0.999 \\
\text { (A) }\end{array}$ & $\begin{array}{l}0.999 \\
\text { (A) }\end{array}$ \\
\hline $\begin{array}{l}\text { M14 } \\
\text { (D) }\end{array}$ & $\begin{array}{l}0.956 \\
\text { (D) }\end{array}$ & $\begin{array}{l}0.956 \\
\text { (C) }\end{array}$ & $\begin{array}{l}0.916 \\
(\mathrm{H})\end{array}$ & $\begin{array}{l}0.936 \\
(\mathrm{~F})\end{array}$ & $\begin{array}{l}0.935 \\
(\mathrm{~F})\end{array}$ & $\begin{array}{l}0.936 \\
(\mathrm{~F})\end{array}$ & $\begin{array}{l}0.879 \\
\text { (E) }\end{array}$ & $\begin{array}{l}0.916 \\
(\mathrm{~F}) \\
\end{array}$ & $\begin{array}{l}0.956 \\
\text { (E) }\end{array}$ & $\begin{array}{l}0.998 \\
\text { (D) }\end{array}$ & $\begin{array}{l}0.998 \\
(\mathrm{C}) \\
\end{array}$ & $\begin{array}{l}0.998 \\
(\mathrm{C}) \\
\end{array}$ & $\begin{array}{l}0.998 \\
\text { (C) }\end{array}$ \\
\hline $\begin{array}{l}\text { M15 } \\
\text { (A) }\end{array}$ & $\begin{array}{l}1.000 \\
\text { (A) }\end{array}$ & $\begin{array}{l}0.996 \\
\text { (A) }\end{array}$ & $\begin{array}{l}0.919 \\
(\mathrm{G})\end{array}$ & $\begin{array}{l}0.960 \\
(\mathrm{C})\end{array}$ & $\begin{array}{l}0.958 \\
(\mathrm{C})\end{array}$ & $\begin{array}{l}0.959 \\
(\mathrm{C})\end{array}$ & $\begin{array}{l}0.919 \\
(\mathrm{C})\end{array}$ & $\begin{array}{l}0.919 \\
\text { (E) }\end{array}$ & $\begin{array}{l}1.000 \\
\text { (A) }\end{array}$ & $\begin{array}{l}0.984 \\
\text { (E) }\end{array}$ & $\begin{array}{l}0.000 \\
(\mathrm{G})\end{array}$ & $\begin{array}{l}0.492 \\
(\mathrm{G})\end{array}$ & $\begin{array}{l}0.000 \\
(\mathrm{G})\end{array}$ \\
\hline
\end{tabular}


Table 10: Results for the models in Table 8 on information measures from divergence group. $\mathrm{S}=$ singularity which cannot be removed. $(\mathrm{R})=$ ranking order for the model, where $\mathrm{R}=\mathrm{A}, \mathrm{B}, \ldots$, in descending order from the top.

\begin{tabular}{|c|c|c|c|c|c|c|c|c|c|c|c|}
\hline Model & $N I_{10}$ & $N I_{11}$ & $N I_{12}$ & $N I_{13}$ & $\mathrm{NI}_{14}$ & $N I_{15}$ & $N I_{16}$ & $N I_{17}$ & $N I_{18}$ & $N I_{19}$ & $\mathrm{NI}_{20}$ \\
\hline $\begin{array}{l}\text { M7 } \\
(\mathrm{F})\end{array}$ & $\begin{array}{l}0.9998 \\
\text { (A) }\end{array}$ & $\begin{array}{l}0.9998 \\
\text { (A) }\end{array}$ & $\begin{array}{l}0.9982 \\
\text { (D) }\end{array}$ & $\begin{array}{l}0.9996 \\
\text { (C) } \\
\end{array}$ & $\begin{array}{l}0.9974 \\
\text { (E) }\end{array}$ & $\begin{array}{l}0.9994 \\
\text { (D) }\end{array}$ & $\begin{array}{l}0.9802 \\
\text { (A) }\end{array}$ & $\begin{array}{l}0.9966 \\
\text { (D) }\end{array}$ & $\begin{array}{l}0.9992 \\
\text { (D) }\end{array}$ & $\begin{array}{l}0.9953 \\
(\mathrm{E})\end{array}$ & $\begin{array}{l}0.9992 \\
\text { (D) }\end{array}$ \\
\hline $\begin{array}{l}\text { M8 } \\
\text { (F) }\end{array}$ & $\begin{array}{l}0.9998 \\
\text { (A) }\end{array}$ & $\begin{array}{l}0.9996 \\
\text { (E) }\end{array}$ & $\begin{array}{l}0.9979 \\
\text { (E) }\end{array}$ & $\begin{array}{l}0.9995 \\
\text { (D) }\end{array}$ & $\begin{array}{l}0.9969 \\
(\mathrm{~F})\end{array}$ & $\begin{array}{l}0.9993 \\
\text { (E) }\end{array}$ & $\begin{array}{l}0.9802 \\
\text { (A) }\end{array}$ & $\begin{array}{l}0.9959 \\
\text { (F) }\end{array}$ & $\begin{array}{l}0.9990 \\
\text { (F) }\end{array}$ & $\begin{array}{l}0.9942 \\
\text { (F) }\end{array}$ & $\begin{array}{l}0.9990 \\
\text { (F) }\end{array}$ \\
\hline $\begin{array}{l}\text { M9 } \\
\text { (C) }\end{array}$ & $\begin{array}{l}0.9998 \\
\text { (A) }\end{array}$ & $\begin{array}{l}0.9996 \\
(\mathrm{E})\end{array}$ & $\begin{array}{l}0.9840 \\
(\mathrm{H})\end{array}$ & $\begin{array}{l}0.9924 \\
\text { (G) }\end{array}$ & $\begin{array}{l}0.9876 \\
\text { (I) }\end{array}$ & $\begin{array}{l}0.9895 \\
(\mathrm{H})\end{array}$ & $\begin{array}{l}0.9802 \\
\text { (A) }\end{array}$ & $\mathrm{S}$ & $\begin{array}{l}0.9893 \\
(\mathrm{H}) \\
\end{array}$ & $\mathrm{S}$ & $\mathrm{S}$ \\
\hline $\begin{array}{l}\text { M10 } \\
\text { (E) }\end{array}$ & $\begin{array}{l}0.9998 \\
\text { (A) }\end{array}$ & $\begin{array}{l}0.9997 \\
\text { (C) }\end{array}$ & $\begin{array}{l}0.9994 \\
\text { (A) }\end{array}$ & $\begin{array}{l}0.9999 \\
\text { (A) }\end{array}$ & $\begin{array}{l}0.9992 \\
\text { (A) }\end{array}$ & $\begin{array}{l}0.9998 \\
\text { (A) }\end{array}$ & $\begin{array}{l}0.9802 \\
\text { (A) }\end{array}$ & $\begin{array}{l}0.9988 \\
\text { (B) }\end{array}$ & $\begin{array}{l}0.9997 \\
\text { (A) }\end{array}$ & $\begin{array}{l}0.9984 \\
\text { (C) }\end{array}$ & $\begin{array}{l}0.9997 \\
\text { (A) }\end{array}$ \\
\hline $\begin{array}{l}\text { M11 } \\
\text { (E) }\end{array}$ & $\begin{array}{l}0.9998 \\
\text { (A) }\end{array}$ & $\begin{array}{l}0.9996 \\
(\mathrm{E})\end{array}$ & $\begin{array}{l}0.9982 \\
\text { (D) }\end{array}$ & $\begin{array}{l}0.9995 \\
\text { (D) }\end{array}$ & $\begin{array}{l}0.9976 \\
\text { (D) }\end{array}$ & $\begin{array}{l}0.9994 \\
\text { (D) }\end{array}$ & $\begin{array}{l}0.9802 \\
\text { (A) }\end{array}$ & $\begin{array}{l}0.9964 \\
(\mathrm{E})\end{array}$ & $\begin{array}{l}0.9991 \\
\text { (E) }\end{array}$ & $\begin{array}{l}0.9950 \\
(\mathrm{~F})\end{array}$ & $\begin{array}{l}0.9991 \\
\text { (E) } \\
\end{array}$ \\
\hline $\begin{array}{l}\text { M12 } \\
\text { (B) }\end{array}$ & $\begin{array}{l}0.9998 \\
\text { (A) }\end{array}$ & $\begin{array}{l}0.9996 \\
(\mathrm{E})\end{array}$ & $\begin{array}{l}0.9852 \\
(\mathrm{G})\end{array}$ & $\begin{array}{l}0.9927 \\
(\mathrm{~F})\end{array}$ & $\begin{array}{l}0.9893 \\
(\mathrm{H})\end{array}$ & $\begin{array}{l}0.9899 \\
\text { (G) }\end{array}$ & $\begin{array}{l}0.9802 \\
\text { (A) }\end{array}$ & $\mathrm{S}$ & $\begin{array}{l}0.9898 \\
(\mathrm{H})\end{array}$ & $\mathrm{S}$ & $\mathrm{S}$ \\
\hline $\begin{array}{l}\text { M13 } \\
\text { (D) }\end{array}$ & $\begin{array}{l}0.9998 \\
\text { (A) }\end{array}$ & $\begin{array}{l}0.9997 \\
\text { (C) }\end{array}$ & $\begin{array}{l}0.9994 \\
\text { (A) }\end{array}$ & $\begin{array}{l}0.9999 \\
\text { (A) }\end{array}$ & $\begin{array}{l}0.9992 \\
\text { (A) }\end{array}$ & $\begin{array}{l}0.9998 \\
\text { (A) }\end{array}$ & $\begin{array}{l}0.9802 \\
\text { (A) }\end{array}$ & $\begin{array}{l}0.9989 \\
\text { (A) }\end{array}$ & $\begin{array}{l}0.9997 \\
\text { (A) }\end{array}$ & $\begin{array}{l}0.9985 \\
\text { (A) }\end{array}$ & $\begin{array}{l}0.9997 \\
\text { (A) }\end{array}$ \\
\hline $\begin{array}{l}\text { M14 } \\
\text { (D) }\end{array}$ & $\begin{array}{l}0.9998 \\
\text { (A) }\end{array}$ & $\begin{array}{l}0.9997 \\
\text { (C) }\end{array}$ & $\begin{array}{l}0.9986 \\
\text { (C) }\end{array}$ & $\begin{array}{l}0.9996 \\
\text { (C) }\end{array}$ & $\begin{array}{l}0.9982 \\
\text { (C) }\end{array}$ & $\begin{array}{l}0.9995 \\
\text { (C) }\end{array}$ & $\begin{array}{l}0.9802 \\
\text { (A) }\end{array}$ & $\begin{array}{l}0.9972 \\
\text { (C) }\end{array}$ & $\begin{array}{l}0.9993 \\
\text { (C) }\end{array}$ & $\begin{array}{l}0.9961 \\
\text { (D) }\end{array}$ & $\begin{array}{l}0.9993 \\
\text { (C) }\end{array}$ \\
\hline $\begin{array}{l}\text { M15 } \\
\text { (A) }\end{array}$ & $\begin{array}{l}0.9998 \\
\text { (A) }\end{array}$ & $\begin{array}{l}0.9998 \\
\text { (A) }\end{array}$ & $\begin{array}{l}0.9856 \\
(\mathrm{~F})\end{array}$ & $\begin{array}{l}0.9928 \\
\text { (E) }\end{array}$ & $\begin{array}{l}0.9899 \\
\text { (G) }\end{array}$ & $\begin{array}{l}0.9900 \\
(\mathrm{~F})\end{array}$ & $\begin{array}{l}0.9802 \\
\text { (A) }\end{array}$ & $S$ & $\begin{array}{l}0.9900 \\
(\mathrm{G})\end{array}$ & $S$ & 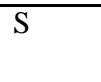 \\
\hline
\end{tabular}

